Research Article

Open Access

Daisuke Kazukawa*

\title{
Concentration of Product Spaces
}

https://doi.org/10.1515/agms-2020-0129

Received July 1, 2020; accepted October 18, 2021

Abstract: We investigate the relation between the concentration and the product of metric measure spaces. We have the natural question whether, for two concentrating sequences of metric measure spaces, the sequence of their product spaces also concentrates. A partial answer is mentioned in Gromov's book [4]. We obtain a complete answer for this question.

Keywords: metric measure space; concentration topology; box topology; product space; metric preserving function

MSC: Primary: 53C23; Secondary: 54E35, 26B35

\section{Introduction}

The geometry and analysis on metric measure spaces have actively been studied. Metric measure spaces typically appear as limit spaces of Riemannian manifolds in the convergence/collapsing theory of Riemannian manifolds. The study of convergence of metric measure spaces is one of central topics in geometric analysis on metric measure spaces. Various convergence notions of metric measure spaces have been defined and studied, such as the measured Gromov-Hausdorff convergence.

Gromov [4, Chapter $3 \frac{1}{2}$ ] has developed a new convergence theory of metric measure spaces based on the concentration of measure phenomenon studied by Lévy and V. Milman $[9,10]$ (see also [8]) which is roughly stated as that any 1-Lipschitz function on high-dimensional spaces is close to a constant. Gromov introduced the observable distance function $d_{\text {conc }}$ on the set of isomorphism classes of metric measure spaces. The observable distance function induces a very characteristic topology, called the concentration topology, which admits the convergence of many sequences whose dimensions are unbounded. Any measured GromovHausdorff convergent sequence concentrates, where we say that a sequence concentrates if it converges in the concentration topology. The study of the concentration topology has been growing in recent years (see $[3,5,14,15,17])$.

In this paper, we study the relation between the concentration topology and the product of metric measure spaces. Gromov has mentioned the concentration of $l_{p}$-product spaces in his theory (see [4, $3 \frac{1}{2}$.46. Example]). The $l_{p}$-product space of two metric measure spaces $X$ and $Y$ for $p \in[1,+\infty]$, write $X \times_{p} Y$, is defined as the Cartesian product set $X \times Y$ equipped with the $l_{p}$-metric $d_{l_{p}}$ and the product measure $m_{X} \otimes m_{Y}$. Gromov's argument implies the following result: for a concentrating sequence $\left\{X_{n}\right\}_{n \in \mathbb{N}}$ and a Lévy family $\left\{Y_{n}\right\}_{n \in \mathbb{N}}$ (i.e., it concentrates to a one-point space), the sequence $\left\{X_{n} \times_{p} Y_{n}\right\}_{n \in \mathbb{N}}$ of their $l_{p}$-product spaces concentrates to the limit space of $\left\{X_{n}\right\}_{n \in \mathbb{N}}$. This gives many examples of sequences of metric measure spaces concentrating to a non-trivial limit space. Our first question is the following: for two sequences $\left\{X_{n}\right\}_{n \in \mathbb{N}}$ and $\left\{Y_{n}\right\}_{n \in \mathbb{N}}$ of metric measure spaces concentrating to metric measure spaces $X$ and $Y$ respectively (both are not necessarily Lévy families), does the sequence $\left\{X_{n} \times_{p} Y_{n}\right\}_{n \in \mathbb{N}}$ of their $l_{p}$-product spaces concentrate to the $l_{p}$-product space $X \times_{p} Y$ of the limit spaces? The main result in this paper gives an affirmative answer to this question. However, its proof is completely different from the original case and is much more complicated.

^Corresponding Author: Daisuke Kazukawa: Department of Mathematics, Osaka University, Toyonaka, Osaka, Japan, E-mail: d-kazukawa@cr.math.sci.osaka-u.ac.jp 
Before describing the main theorem, we explain a notion of product, which is a generalized notion of the $l_{p}$-product, of two metric measure spaces. Let $F:[0,+\infty)^{2} \rightarrow[0,+\infty)$ be a continuous function satisfying the following condition: for any two metric spaces $\left(X, d_{X}\right)$ and $\left(Y, d_{Y}\right)$, the function

$$
d_{F}\left((x, y),\left(x^{\prime}, y^{\prime}\right)\right):=F\left(d_{X}\left(x, x^{\prime}\right), d_{Y}\left(y, y^{\prime}\right)\right)
$$

is a metric on $X \times Y$. Such a function $F$ is called a metric preserving function. Given two metric measure spaces $X, Y$ and a continuous metric preserving function $F:[0,+\infty)^{2} \rightarrow[0,+\infty)$, the triple

$$
\left(X \times Y, d_{F}, m_{X} \otimes m_{Y}\right)
$$

is a metric measure space. In this paper, this space is called the product space of $X$ and $Y$ generated by $F$ and is denoted by $X \times_{F} Y$. For any extended real number $p \in[1,+\infty]$, the function

$$
F_{p}(s, t):= \begin{cases}\left(s^{p}+t^{p}\right)^{\frac{1}{p}} & \text { if } p<+\infty, \\ \max \{s, t\} & \text { if } p=+\infty\end{cases}
$$

is a metric preserving function. The distance function $d_{F_{p}}$ coincides with the $l_{p}$-metric $d_{l_{p}}$, and then the product space $X \times_{F_{p}} Y$ coincides with the $l_{p}$-product space $X \times_{p} Y$. Thus, the product generated by the metric preserving functions is a generalization of the $l_{p}$-product. Other than the function $F_{p}$, for example, the two functions

$$
\log \left(e^{s}+e^{t}-1\right), \quad \frac{1}{2}(s+t)+\frac{1}{2} \max \{s, t\}
$$

are two of the easiest examples of metric preserving functions. However, general metric preserving functions are more complicated. We say that the function $F$ is an isotone if $F(s, t) \leq F\left(s^{\prime}, t^{\prime}\right)$ for all $s \leq s^{\prime}$ and $t \leq t^{\prime}$. In general, metric preserving functions are not necessarily isotones. We describe some properties of product spaces in Section 3.1 and show a lot more examples of metric preserving functions in Section 3.2.

The following main theorem gives an answer to the more general question than that stated before for the $l_{p}$-product.

Theorem 1.1. Let $F_{n}, F:[0,+\infty)^{2} \rightarrow[0,+\infty), n=1,2, \ldots$, be continuous metric preserving functions. Assume that $F_{n}$ converges pointwise to $F$ as $n \rightarrow \infty$. Then the following (1) and (2) are equivalent to each other.

(1) For any two sequences $\left\{X_{n}\right\}_{n \in \mathbb{N}}$ and $\left\{Y_{n}\right\}_{n \in \mathbb{N}}$ of metric measure spaces concentrating to metric measure spaces $X$ and $Y$ respectively, the sequence $\left\{X_{n} \times_{F_{n}} Y_{n}\right\}_{n \in \mathbb{N}}$ of their product spaces concentrates to the product space $X \times_{F} Y$ as $n \rightarrow \infty$.

(2) For any $s, t \in[0,+\infty)$,

$$
\lim _{n \rightarrow \infty}\left(F_{n}(s, t)-\inf _{s \leq s^{\prime} ; t \leq t^{\prime}} F_{n}\left(s^{\prime}, t^{\prime}\right)\right)=0
$$

Remark 1.2. We set

$$
I_{n}(s, t):=F_{n}(s, t)-\inf _{s \leq s^{\prime} ; t \leq t^{\prime}} F_{n}\left(s^{\prime}, t^{\prime}\right)
$$

and consider the following five conditions.

(i) The functions $F_{n}$ are isotones (i.e., $I_{n} \equiv 0$ ) for all $n \in \mathbb{N}$.

(ii) $\lim _{n \rightarrow \infty} \sup _{s, t \geq 0} I_{n}(s, t)=0$.

(iii) $\lim _{n \rightarrow \infty} \sup _{0 \leq s, t \leq D} I_{n}(s, t)=0$ for any $D>0$.

(iv) $\lim _{n \rightarrow \infty} I_{n}(s, t)=0$ for any $s, t \in[0,+\infty)$.

(v) The function $F$ is an isotone.

Under the setting of Theorem 1.1, it is easy to see that (i) $\Rightarrow$ (ii) $\Rightarrow$ (iii) $\Rightarrow$ (iv) $\Rightarrow$ (v). On the other hand, we see that only (iv) $\Rightarrow$ (iii) holds among the converse implications (see Lemmas 4.6, 4.10 and Example 3.18). The condition (iii) is also equivalent to the concentration of product spaces, that is, the condition (1) of Theorem 1.1.

Since the function $F_{p}$ is an isotone for all $p \in[1,+\infty]$, we obtain the following corollary. 
Corollary 1.3. Let $\left\{X_{n}\right\}_{n \in \mathbb{N}}$ and $\left\{Y_{n}\right\}_{n \in \mathbb{N}}$ be two sequences of metric measure spaces concentrating to metric measure spaces $X$ and $Y$ respectively. Assume that $p_{n} \in[1,+\infty]$ converges to $p \in[1,+\infty]$ as $n \rightarrow \infty$. Then the sequence $\left\{X_{n} \times_{p_{n}} Y_{n}\right\}_{n \in \mathbb{N}}$ of their $l_{p_{n}}$-product spaces concentrates to the $l_{p}$-product space $X \times_{p} Y$ as $n \rightarrow \infty$.

We prove Theorem 1.1 in Section 4.2 and Section 5. In addition, from our main result, we construct new examples of sequences of metric measure spaces concentrating to a non-trivial limit space. It was difficult to construct a new example of concentrating sequences and this point was a serious problem for the study of concentration. We describe in Section 4.3 a new specific example of the concentrating sequences that are obtained by Theorem 1.1.

Furthermore, it is interesting to compare Theorem 1.1 with Proposition 4.2. This proposition shows that the convergence of product spaces in the box topology is true unconditionally. The box topology is finer than the concentration topology and had been introduced by Gromov at the same time as the concentration topology. A relation between the box topology and the product spaces is summarized in Section 4.1.

As another topic, the notion of metric preserving functions gives another question. Let $F:[0,+\infty) \rightarrow$ $[0,+\infty)$ be a function such that for any metric space $\left(X, d_{X}\right)$, the function $F \circ d_{X}$ is a metric on $X$. This $F$ is also called a metric preserving function. Roughly speaking, such a function is a 1-dimensional version of a metric preserving function defined before. We consider the following question: does a metric-transformed sequence of a concentrating sequence by some metric preserving functions also concentrate? We obtain an answer, which is related with Theorem 1.1.

Theorem 1.4. Let $F_{n}, F:[0,+\infty) \rightarrow[0,+\infty), n=1,2, \ldots$, be continuous metric preserving functions. Assume that $F_{n}$ converges pointwise to $F$ as $n \rightarrow \infty$. Then the following (1) and (2) are equivalent to each other.

(1) For any sequence $\left\{X_{n}\right\}_{n \in \mathbb{N}}$ of metric measure spaces concentrating to a metric measure space $X$, the sequence $\left\{\left(X_{n}, F_{n} \circ d_{X_{n}}, m_{X_{n}}\right)\right\}_{n \in \mathbb{N}}$ concentrates to $\left(X, F \circ d_{X}, m_{X}\right)$ as $n \rightarrow \infty$.

(2) For any $s \in[0,+\infty)$,

$$
\lim _{n \rightarrow \infty}\left(F_{n}(s)-\inf _{s \leq s^{\prime}} F_{n}\left(s^{\prime}\right)\right)=0 .
$$

The implication “(2) $\Rightarrow$ (1)" of Theorem 1.4 is a corollary of Theorem 1.1. On the other hand, in Section 5, the proof of "(1) $\Rightarrow$ (2)" of Theorem 1.4 gives an essential idea to the proof of Theorem 1.1. We prove this in Section 4.2 and Section 5 together with Theorem 1.1.

As a matter of fact, we are able to generalize Theorem 1.1 to a statement for product spaces of $N$ metric measure spaces for any finite number $N$. This generalization is shown in Section 6.

\section{Preliminaries}

In this section, we describe the definitions and some properties of metric measure space, the box distance and the observable distance. We use most of these notions along [17]. As for more details, we refer to [17] and $\left[4\right.$, Chapter $3 \frac{1}{2}+$.

\subsection{Metric measure spaces}

Let $\left(X, d_{X}\right)$ be a complete separable metric space and $m_{X}$ a Borel probability measure on $X$. We call the triple $\left(X, d_{X}, m_{X}\right)$ a metric measure space, or an $m m$-space for short. We sometimes say that $X$ is an mm-space, in which case the metric and the measure of $X$ are respectively indicated by $d_{X}$ and $m_{X}$.

Definition 2.1 (mm-Isomorphism). Two mm-spaces $X$ and $Y$ are said to be $m m$-isomorphic to each other if there exists an isometry $f: \operatorname{supp} m_{X} \rightarrow \operatorname{supp} m_{Y}$ such that $f_{\star} m_{X}=m_{Y}$, where $f_{\star} m_{X}$ is the push-forward 
measure of $m_{X}$ by $f$. Such an isometry $f$ is called an mm-isomorphism. Denote by $X$ the set of $\mathrm{mm}$-isomorphism classes of mm-spaces.

Note that an mm-space $X$ is mm-isomorphic to ( $\operatorname{supp} m_{X}, d_{X}, m_{X}$ ). We assume that an mm-space $X$ satisfies $X=\operatorname{supp} m_{X}$ unless otherwise stated.

\subsection{Observable diameter}

For a metric space $\left(X, d_{X}\right)$, we denote by $\mathcal{L} i p_{1}(X)$ the set of 1-Lipschitz functions on $X$.

The observable diameter is one of the most fundamental invariants of an mm-space.

Definition 2.2 (Partial and observable diameter). Let $X$ be an mm-space. For a real number $\alpha \leq 1$, we define the partial diameter $\operatorname{diam}(X ; \alpha)$ of $X$ to be the infimum of $\operatorname{diam} A$, where $A \subset X$ runs over all Borel subsets with $m_{X}(A) \geq \alpha$ and $\operatorname{diam} A$ is the diameter of $A$. For a real number $\kappa>0$, we define the observable diameter of $X$ to be

$$
\operatorname{ObsDiam}(X ;-\kappa):=\sup _{f \in \mathcal{L} i p_{1}(X)} \operatorname{diam}\left(\left(\mathbb{R},|\cdot|, f_{\star} m_{X}\right) ; 1-\kappa\right)
$$

The observable diameter is an invariant under mm-isomorphism. Note that $\operatorname{ObsDiam}(X ;-\kappa)$ is nonincreasing in $\kappa>0$.

Definition 2.3 (Lévy family). A sequence of mm-spaces $\left\{X_{n}\right\}_{n \in \mathbb{N}}$ is called a Lévy family if

$$
\lim _{n \rightarrow \infty} \operatorname{ObsDiam}\left(X_{n} ;-\kappa\right)=0
$$

for any $\kappa>0$.

\subsection{Box distance and observable distance}

For a subset $A$ of a metric space $\left(X, d_{X}\right)$ and for a real number $r>0$, we set

$$
U_{r}(A):=\left\{x \in X \mid d_{X}(x, A)<r\right\}
$$

where $d_{X}(x, A):=\inf _{a \in A} d_{X}(x, a)$.

Definition 2.4 (Prokhorov distance). Let $\lambda>0$ be a real number. The $\lambda$-Prokhorov distance $d_{\mathrm{P}}^{(\lambda)}(\mu, v)$ between two Borel probability measures $\mu$ and $v$ on a metric space $X$ is defined to be the infimum of $\varepsilon>0$ satisfying

$$
\mu\left(U_{\varepsilon}(A)\right) \geq v(A)-\lambda \varepsilon
$$

for any Borel subset $A \subset X$. In particular, the 1-Prokhorov distance $d_{\mathrm{P}}^{(1)}$ is called the Prokhorov distance and we denote it by $d_{\mathrm{P}}$.

The Prokhorov metric $d_{\mathrm{P}}$ is a metrization of the weak convergence of Borel probability measures on $X$ provided that $X$ is a separable metric space.

Definition 2.5 (Ky Fan metric). Let $(X, \mu)$ be a measure space and $\left(Y, d_{Y}\right)$ a metric space. For two $\mu$ measurable maps $f, g: X \rightarrow Y$, we define $d_{\mathrm{KF}}^{\mu}(f, g)$ to be the infimum of $\varepsilon \geq 0$ satisfying

$$
\mu\left(\left\{x \in X \mid d_{Y}(f(x), g(x))>\varepsilon\right\}\right) \leq \varepsilon .
$$

The two variable function $d_{\mathrm{KF}}^{\mu}$ is a metric on the set of $\mu$-measurable maps from $X$ to $Y$ by identifying two maps if they are equal to each other $\mu$-almost everywhere. We call $d_{\mathrm{KF}}^{\mu}$ the Ky Fan metric. 
Lemma 2.6 ([17, Lemma 1.26]). Let $X$ be a topological space with a Borel probability measure $\mu$ and $Y$ a metric space. For any two Borel measurable maps $f, g: X \rightarrow Y$, we have

$$
d_{\mathrm{P}}\left(f_{\star} \mu, g_{\star} \mu\right) \leq d_{\mathrm{KF}}^{\mu}(f, g) .
$$

Definition 2.7 (Parameter). Let $I:=[0,1)$ and let $X$ be an mm-space. A map $\varphi: I \rightarrow X$ is called a parameter of $X$ if $\varphi$ is a Borel measurable map such that

$$
\varphi_{\star} \mathcal{L}^{1}=m_{X},
$$

where $\mathcal{L}^{1}$ is the one-dimensional Lebesgue measure on $I$.

Lemma 2.8 ([17, Lemma 4.2]). Any mm-space has a parameter.

Definition 2.9 (Box distance). We define the box distance $\square(X, Y)$ between two mm-spaces $X$ and $Y$ to be the infimum of $\varepsilon \geq 0$ satisfying that there exist parameters $\varphi: I \rightarrow X, \psi: I \rightarrow Y$, and a Borel subset $I_{0} \subset I$ with $\mathcal{L}^{1}\left(I_{0}\right) \geq 1-\varepsilon$ such that

$$
\left|d_{X}(\varphi(s), \varphi(t))-d_{Y}(\psi(s), \psi(t))\right| \leq \varepsilon
$$

for any $s, t \in I_{0}$.

Theorem 2.10 ([17, Theorem 4.10]). The box distance function $\square$ is a complete separable metric on $X$.

Lemma 2.11 ([17, Proposition 4.12]). Let $X$ be a complete separable metric space. For any two Borel probability measures $\mu$ and $v$ on $X$, we have

$$
\square((X, \mu),(X, v)) \leq 2 d_{\mathrm{P}}(\mu, v) .
$$

The following notion gives one of the conditions that are equivalent to the box convergence.

Definition 2.12 ( $\varepsilon$-mm-Isomorphism). Let $X$ and $Y$ be two mm-spaces and $f: X \rightarrow Y$ a Borel measurable map. Let $\varepsilon \geq 0$ be a real number. We say that $f$ is an $\varepsilon$-mm-isomorphism if there exists a Borel subset $X_{0} \subset X$ such that

(1) $m_{X}\left(X_{0}\right) \geq 1-\varepsilon$,

(2) $\left|d_{X}\left(x, x^{\prime}\right)-d_{Y}\left(f(x), f\left(x^{\prime}\right)\right)\right| \leq \varepsilon$ for any $x, x^{\prime} \in X_{0}$,

(3) $d_{\mathrm{P}}\left(f_{\star} m_{X}, m_{Y}\right) \leq \varepsilon$

We call $X_{0}$ a nonexceptional domain of $f$.

It is easy to see that, for a 0 -mm-isomorphism $f: X \rightarrow Y$, there is an mm-isomorphism $\hat{f}: X \rightarrow Y$ that is equal to $f m_{X}$-a.e. on $X$.

Lemma 2.13 ([17, Lemma 4.22]).

(1) If there exists an $\varepsilon$-mm-isomorphism $f: X \rightarrow Y$, then $\square(X, Y) \leq 3 \varepsilon$.

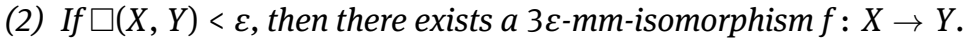

For any topological space $X$, any metric space $Y$, and any Borel measurable map $p: X \rightarrow Y$, we set

$$
p^{\star} \operatorname{Lip}_{1}(Y):=\left\{f \circ p \mid f \in \mathcal{L i p}_{1}(Y)\right\} .
$$

Note that, for any mm-space $X$ and any parameter $\varphi: I \rightarrow X$ of $X$, the set $\varphi^{\star} \mathcal{L} \operatorname{ip}_{1}(X)$ consists of Borel measurable functions on $I$.

Definition 2.14 (Observable distance). We define the observable distance $d_{\text {conc }}(X, Y)$ between two $m m$-spaces $X$ and $Y$ by

$$
d_{\text {conc }}(X, Y):=\inf _{\varphi, \psi} d_{\mathrm{H}}\left(\varphi^{\star} \mathcal{L} i p_{1}(X), \psi^{\star} \mathcal{L} i p_{1}(Y)\right),
$$


where $\varphi: I \rightarrow X$ and $\psi: I \rightarrow Y$ run over all parameters of $X$ and $Y$ respectively, and $d_{\mathrm{H}}$ is the Hausdorff distance with respect to the metric $d_{\mathrm{KF}}^{\mathcal{L}^{1}}$. We say that a sequence of mm-spaces $\left\{X_{n}\right\}_{n \in \mathbb{N}}$ concentrates to an

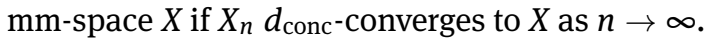

Proposition 2.15 ([17, Corollary 5.8]). Let $\left\{X_{n}\right\}_{n \in \mathbb{N}}$ be a sequence ofmm-spaces. Then, $\left\{X_{n}\right\}_{n \in \mathbb{N}}$ is a Lévy family if and only if $\left\{X_{n}\right\}_{n \in \mathbb{N}}$ concentrates to a one-point mm-space as $n \rightarrow \infty$.

Example 2.16 ([17, Section 2.3]). Let $S^{n}\left(r_{n}\right), n=1,2, \ldots$, be the sphere of radius $r_{n}>0$ in $\mathbb{R}^{n+1}$. Assume that $S^{n}\left(r_{n}\right)$ is endowed with the standard Riemannian metric. Let $\sigma^{n}$ be the Riemannian volume measure on $S^{n}\left(r_{n}\right)$ normalized as $\sigma^{n}\left(S^{n}\left(r_{n}\right)\right)=1$. Then we have

$$
\operatorname{ObsDiam}\left(\left(S^{n}\left(r_{n}\right), d_{S^{n}\left(r_{n}\right)}, \sigma^{n}\right) ;-\kappa\right)=O\left(r_{n} n^{-1 / 2}\right)
$$

for any $\kappa>0$ as $n \rightarrow \infty$. That is, the sequence $\left\{S^{n}\left(r_{n}\right)\right\}_{n \in \mathbb{N}}$ is a Lévy family if $r_{n}=o(\sqrt{n})$.

Proposition 2.17 ([17, Proposition 5.5]). For any two $m m$-spaces $X$ and $Y$, we have

$$
d_{\text {conc }}(X, Y) \leq \square(X, Y) .
$$

Definition 2.18 (Enforce $\varepsilon$-concentration). A Borel measurable map $p: X \rightarrow Y$ is said to enforce $\varepsilon$ concentration of $X$ to $Y$ if

$$
d_{\mathrm{H}}\left(\mathcal{L i p}_{1}(X), p^{*} \operatorname{Lip}_{1}(Y)\right) \leq \varepsilon,
$$

where $d_{\mathrm{H}}$ is the Hausdorff distance with respect to the metric $d_{\mathrm{KF}}^{m_{X}}$.

Theorem 2.19 ([17, Corollary 5.36]). Let $X_{n}$ and $X$ be mm-spaces, where $n=1,2, \ldots$. Then the following (1) and (2) are equivalent to each other.

(1) $\left\{X_{n}\right\}_{n \in \mathbb{N}}$ concentrates to $X$ as $n \rightarrow \infty$.

(2) There exists a sequence of Borel measurable maps $p_{n}: X_{n} \rightarrow X, n=1,2, \ldots$, that enforce $\varepsilon_{n}$-concentration of $X_{n}$ to $X$ and $d_{\mathrm{P}}\left(\left(p_{n}\right)_{\star} m_{X_{n}}, m_{X}\right) \leq \varepsilon_{n}$ for all $n$ and for some sequence $\varepsilon_{n} \rightarrow 0$.

\subsection{Strassen's theorem and Lévy mean}

Definition 2.20 (Transport plan). Let $\mu$ and $v$ be two finite Borel measures on $X$. A Borel measure $\pi$ on $X \times X$ is called a transport plan (or coupling) between $\mu$ and $v$ if

$$
\pi(A \times X)=\mu(A) \text { and } \pi(X \times A)=v(A)
$$

for any Borel subset $A \subset X$.

Definition 2.21 ( $\varepsilon$-Subtransport plan). Let $\mu$ and $v$ be two Borel probability measures on $X$. A Borel measure $\pi$ on $X \times X$ is called an $\varepsilon$-subtransport plan between $\mu$ and $v$ if there exist two Borel measures $\mu^{\prime}$ and $v^{\prime}$ on $X$ with $\mu^{\prime} \leq \mu$ and $\nu^{\prime} \leq \nu$ such that $\pi$ is a transport plan between $\mu^{\prime}$ and $v^{\prime}$, and $\pi$ satisfies

$$
\operatorname{supp} \pi \subset \Delta_{\varepsilon}:=\left\{\left(x, x^{\prime}\right) \in X \times X \mid d_{X}\left(x, x^{\prime}\right) \leq \varepsilon\right\} .
$$

For an $\varepsilon$-subtransport plan $\pi$ between $\mu$ and $v$, the deficiency of $\pi$ is defined to be

$$
\operatorname{def} \pi:=1-\pi(X \times X) .
$$

Theorem 2.22 (Strassen's theorem). Assume that $X$ is a complete separable metric space. For any real number $\lambda>0$ and for any two Borel probability measures $\mu$ and $v$ on $X$, we have

$$
d_{\mathrm{P}}^{(\lambda)}(\mu, v)=\inf \left\{\begin{array}{l|l}
\varepsilon>0 & \begin{array}{l}
\text { There exists an } \varepsilon \text {-subtransport plan } \pi \\
\text { between } \mu \text { and } v \text { with def } \pi \leq \lambda \varepsilon
\end{array}
\end{array}\right\} .
$$


Definition 2.23 (Median and Lévy mean). Let $X$ be a measure space with probability measure $\mu$ and $f: X \rightarrow \mathbb{R}$ a measurable function. A real number $m \in \mathbb{R}$ is called a median of $f$ if it satisfies

$$
\mu(\{x \in X \mid f(x) \geq m\}) \geq \frac{1}{2} \text { and } \mu(\{x \in X \mid f(x) \leq m\}) \geq \frac{1}{2} .
$$

It is easy to see that the set of medians of $f$ is a closed and bounded interval. The Lévy mean $\operatorname{lm}(f ; \mu)$ of $f$ with respect to $\mu$ is defined to be

$$
\operatorname{lm}(f ; \mu):=\frac{\underline{m}+\bar{m}}{2}
$$

where $\underline{m}$ is the minimum of medians of $f$, and $\bar{m}$ the maximum of medians of $f$.

Proposition 2.24 ([17, Section 2.3]). Let $\left\{X_{n}\right\}_{n \in \mathbb{N}}$ be a sequence of mm-spaces. Then, $\left\{X_{n}\right\}_{n \in \mathbb{N}}$ is a Lévy family if and only if for any $f_{n} \in \mathcal{L} i p_{1}\left(X_{n}\right)$,

$$
\lim _{n \rightarrow \infty} d_{\mathrm{KF}}^{m_{X_{n}}}\left(f_{n}, \operatorname{lm}\left(f_{n} ; m_{X_{n}}\right)\right)=0 .
$$

Definition 2.25 (Lévy radius). Let $X$ be an mm-space and $\kappa>0$ a real number. The Lévy radius LeRad $(X ;-\kappa)$ of $X$ is defined to be the infimum of $\varepsilon>0$ satisfying

$$
m_{X}\left(\left\{x \in X|| f(x)-\operatorname{lm}\left(f ; m_{X}\right) \mid>\varepsilon\right\}\right) \leq \kappa
$$

for any $f \in \mathcal{L i p}_{1}(X)$.

Lemma 2.26 ([17, Lemma 7.31]). Let $X$ be an $m m$-space. For any $\kappa$ with $0<\kappa<1 / 2$, we have

$$
\operatorname{LeRad}(X ;-\kappa) \leq \operatorname{ObsDiam}(X ;-\kappa) .
$$

Lemma 2.27 ([17, Lemma 9.6]). Let $\mu$ and $v$ be two Borel probability measures on a metric space $X$. Assume that there exists an $\varepsilon$-subtransport plan $\pi$ between $\mu$ and $v$ with def $\pi<1-2 \kappa$ for two real numbers $\varepsilon$ and $\kappa$ with $\varepsilon>0$ and $0<\kappa<1 / 2$. Then, for any 1-Lipschitz function $f: X \rightarrow \mathbb{R}$, we have

$$
|\operatorname{lm}(f ; \mu)-\operatorname{lm}(f ; v)| \leq \varepsilon+\operatorname{ObsDiam}((X, \mu) ;-\kappa)+\operatorname{ObsDiam}((X, v) ;-\kappa) .
$$

\section{Product space of metric measure spaces}

\subsection{Metric preserving functions and product spaces}

Definition 3.1 (Metric preserving function). Let $N \in \mathbb{N}$. A function $F:[0,+\infty)^{N} \rightarrow[0,+\infty)$ is called a metric preserving function if for any $N$ metric spaces $\left(X_{1}, d_{X_{1}}\right), \ldots,\left(X_{N}, d_{X_{N}}\right)$, the function

$$
d_{F}\left(\left(x_{i}\right)_{i=1}^{N},\left(x_{i}^{\prime}\right)_{i=1}^{N}\right):=F\left(d_{X_{1}}\left(x_{1}, x_{1}^{\prime}\right), \ldots, d_{X_{N}}\left(x_{N}, x_{N}^{\prime}\right)\right)
$$

is a metric on $X_{1} \times \cdots \times X_{N}$.

Note that for a metric preserving function $F$,

$$
F^{-1}(0)=\{(0, \ldots, 0)\}
$$

holds necessarily. Let $a, b$, and $c$ be three nonnegative real numbers. We call the triplet $(a, b, c)$ a triangle triplet if $a \leq b+c, b \leq a+c$, and $c \leq a+b$ are all satisfied.

Theorem 3.2 ([1, Theorem 2.6]). A function $F:[0,+\infty)^{N} \rightarrow[0,+\infty)$ with $F^{-1}(0)=\{(0, \ldots, 0)\}$ is a metric preserving function if and only if for any $N$ triangle triplets $\left(a_{i}, b_{i}, c_{i}\right), i=1, \ldots, N$, the triplet

$$
\left(F\left(a_{1}, \ldots, a_{N}\right), F\left(b_{1}, \ldots, b_{N}\right), F\left(c_{1}, \ldots, c_{N}\right)\right)
$$

is a triangle triplet. 
Corollary 3.3. Let $F:[0,+\infty)^{N} \rightarrow[0,+\infty)$ be a metric preserving function. Then, for any $\left(s_{i}\right)_{i=1}^{N},\left(s_{i}^{\prime}\right)_{i=1}^{N} \in$ $[0,+\infty)^{N}$, we have

(1) $\left|F\left(s_{1}, \ldots, s_{N}\right)-F\left(s_{1}^{\prime}, \ldots, s_{N}^{\prime}\right)\right| \leq F\left(\left|s_{1}-s_{1}^{\prime}\right|, \ldots,\left|s_{N}-s_{N}^{\prime}\right|\right)$,

(2) $F\left(s_{1}, \ldots, s_{N}\right) \leq 2 F\left(s_{1}^{\prime}, \ldots, s_{N}^{\prime}\right)$ if $s_{i} \leq 2 s_{i}^{\prime}$ for every $i$.

Proof. For any $s, s^{\prime} \in[0,+\infty)$, the triplet $\left(s, s^{\prime},\left|s-s^{\prime}\right|\right)$ is a triangle triplet, and the triplet $\left(s, s^{\prime}, s^{\prime}\right)$ is a triangle triplet if $s \leq 2 s^{\prime}$. Applying Theorem 3.2 to them, we obtain this corollary.

Definition 3.4. Let $N \in \mathbb{N}$. A function $F:[0,+\infty)^{N} \rightarrow[0,+\infty)$ is said to be subadditive if for any $\left(s_{i}\right)_{i=1}^{N},\left(s_{i}^{\prime}\right)_{i=1}^{N} \in[0,+\infty)^{N}$,

$$
F\left(s_{1}+s_{1}^{\prime}, \ldots, s_{N}+s_{N}^{\prime}\right) \leq F\left(s_{1}, \ldots, s_{N}\right)+F\left(s_{1}^{\prime}, \ldots, s_{N}^{\prime}\right) .
$$

$F$ is called an isotone if

$$
F\left(s_{1}, \ldots, s_{N}\right) \leq F\left(s_{1}^{\prime}, \ldots, s_{N}^{\prime}\right)
$$

for any $\left(s_{i}\right)_{i=1}^{N},\left(s_{i}^{\prime}\right)_{i=1}^{N} \in[0,+\infty)^{N}$ such that $s_{i} \leq s_{i}^{\prime}$ for each $i$.

In the case of $N=1$, an isotone means a nondecreasing function.

Lemma 3.5 ([6, Exercise 4.C], [12, Satz 1]). Let $N \in \mathbb{N}$ and let $F:[0,+\infty)^{N} \rightarrow[0,+\infty)$ be a function. Then the following (1) and (2) hold.

(1) If $F$ is a metric preserving function, then $F$ is subadditive.

(2) If $F$ is a subadditive isotone fulfilling $F^{-1}(0)=\{(0, \ldots, 0)\}$, then $F$ is a metric preserving function.

Remark 3.6. There are many examples of metric preserving functions that are not isotones even if $N=1$. We show such examples in the next subsection.

In this paper, we usually consider the case of $N=2$.

Example 3.7. We cite some easy (however important) examples of metric preserving functions.

(1) For any extended real number $p \in[1,+\infty]$, we define

$$
F_{p}(s, t):= \begin{cases}\left(s^{p}+t^{p}\right)^{\frac{1}{p}} & \text { if } p<+\infty \\ \max \{s, t\} & \text { if } p=+\infty .\end{cases}
$$

(2) Let $F_{\exp }(s, t):=\log \left(e^{s}+e^{t}-1\right)$.

(3) For any real number $\alpha \in(0,1)$, we define

$$
F_{\alpha}(s, t):=s^{\alpha}+t^{\alpha}
$$

(4) For two real numbers $p, q \in[1,+\infty)$ with $p<q$, we define

$$
F_{p, q}(s, t):=\left(s^{p}+t^{p}\right)^{\frac{1}{q}}
$$

Theorem $3.8([1$, Section 3$])$. Let $F:[0,+\infty)^{N} \rightarrow[0,+\infty)$ be a metric preserving function. Then the following conditions are equivalent to each other.

(1) $F$ is continuous.

(2) $F$ is continuous at $(0, \ldots, 0)$.

(3) F is uniformly continuous.

(4) For each $i=1, \ldots, N$, the map $F \circ \iota_{i}:[0,+\infty) \rightarrow[0,+\infty)$ is continuous, where $\iota_{i}:[0,+\infty) \rightarrow[0,+\infty)^{N}$ is the natural $i$-th inclusion map (i.e., $\operatorname{pr}_{i} \circ \iota_{i}=\mathrm{id}$ and $\mathrm{pr}_{j} \circ \iota_{i}=0$ for $j \neq i$ ).

(5) For any $N$ metric spaces $\left(X_{1}, d_{X_{1}}\right), \ldots,\left(X_{N}, d_{X_{N}}\right)$, the metric $d_{F}$ induces the product topology on $X_{1} \times \cdots \times X_{N}$. 
Remark 3.9. In the case of $N=1$, we understand the condition (5) in the above theorem to mean that $\left(X, F \circ d_{X}\right)$ has the same topology as $\left(X, d_{X}\right)$.

Proposition 3.10. Let $F:[0,+\infty)^{N} \rightarrow[0,+\infty)$ be a continuous metric preserving function. If $N$ metric spaces $X_{1}, \ldots, X_{N}$ are complete, then the metric space $\left(X_{1} \times \cdots \times X_{N}, d_{F}\right)$ is also complete.

Proof. We take any $d_{F}$-Cauchy sequence $\left\{\left(x_{n}^{1}, \ldots, x_{n}^{N}\right)\right\}_{n \in \mathbb{N}}$. We have

$$
\lim _{m, n \rightarrow \infty} F\left(d_{X_{1}}\left(x_{m}^{1}, x_{n}^{1}\right), \ldots, d_{X_{N}}\left(x_{m}^{N}, x_{n}^{N}\right)\right)=0 .
$$

Fix $i \in\{1, \ldots, N\}$. Since

$$
F\left(0, \ldots, 0, d_{X_{i}}\left(x_{m}^{i}, x_{n}^{i}\right), 0, \ldots, 0\right) \leq 2 F\left(d_{X_{1}}\left(x_{m}^{1}, x_{n}^{1}\right), \ldots, d_{X_{N}}\left(x_{m}^{N}, x_{n}^{N}\right)\right) \rightarrow 0
$$

as $m, n \rightarrow \infty$, Corollary 3.3 (2) leads to

$$
\lim _{m, n \rightarrow \infty} d_{X_{i}}\left(x_{m}^{i}, x_{n}^{i}\right)=0 .
$$

By the completeness of $X_{i}$, there exists $x^{i} \in X_{i}$ such that $x_{n}^{i} \rightarrow x^{i}$ in $X_{i}$ as $n \rightarrow \infty$. Thus

$$
\lim _{n \rightarrow \infty} d_{F}\left(\left(x_{n}^{1}, \ldots, x_{n}^{N}\right),\left(x^{1}, \ldots, x^{N}\right)\right)=\lim _{n \rightarrow \infty} F\left(d_{X_{1}}\left(x_{n}^{1}, x^{1}\right), \ldots, d_{X_{N}}\left(x_{n}^{N}, x^{N}\right)\right)=0,
$$

which implies that $d_{F}$ is complete.

We denote by $\mathscr{F}^{N}$ the set of continuous metric preserving functions on $[0,+\infty)^{N}$.

Definition 3.11 (Product space). Given two mm-spaces $X, Y$ and a function $F \in \mathscr{F}^{2}$, we define the product space $X \times_{F} Y$ of $X$ and $Y$ as the mm-space

$$
\left(X \times Y, d_{F}, m_{X} \otimes m_{Y}\right)
$$

which consists of the complete separable metric space $\left(X \times Y, d_{F}\right)$ and the product measure $m_{X} \otimes m_{Y}$ of $m_{X}$ and $m_{Y}$.

Definition 3.12 ( $l_{p}$-Product space). For two mm-spaces $X, Y$ and an extended real number $p \in[1,+\infty]$, we call the distance function $d_{F_{p}}$ on $X \times Y$, where $F_{p}$ is of Example 3.7 (1), the $l_{p}$-metric, and the product space $X \times_{F_{p}} Y$ the $l_{p}$-product space. From now on, we denote $X \times_{F_{p}} Y$ by $X \times_{p} Y$.

\subsection{Examples of metric preserving function}

In this subsection, we present many examples of continuous metric preserving functions. At first, we are able to make such functions from the Mulholland inequality known as the generalized Minkowski inequality.

Theorem 3.13 (Mulholland inequality [11], [7, Theorem 8.8.1]). If both a homeomorphism $\varphi:[0,+\infty) \rightarrow$ $[0,+\infty)$ (i.e., continuous and increasing bijection with $\varphi(0)=0)$ and $\log \circ \varphi \circ \exp$ are convex, then

$$
\varphi^{-1}\left(\varphi\left(s+s^{\prime}\right)+\varphi\left(t+t^{\prime}\right)\right) \leq \varphi^{-1}(\varphi(s)+\varphi(t))+\varphi^{-1}\left(\varphi\left(s^{\prime}\right)+\varphi\left(t^{\prime}\right)\right)
$$

for any $s, s^{\prime}, t, t^{\prime} \in[0,+\infty)$.

For a homeomorphism $\varphi:[0,+\infty) \rightarrow[0,+\infty)$, the function $F_{\varphi}$ on $[0,+\infty)^{2}$ defined by

$$
F_{\varphi}(s, t):=\varphi^{-1}(\varphi(s)+\varphi(t))
$$

for $s, t \in[0,+\infty)$ is an isotone with $F^{-1}(0)=\{(0,0)\}$. The Mulholland inequality says that if both $\varphi$ and $\log \circ \varphi \circ \exp$ are convex, then $F_{\varphi}$ is subadditive, so that it is a metric preserving function. 
Lemma 3.14 ([7, Lemma 8.8.1]). We consider the following two classes $A$ and $B$ of functions.

(A) The class A consists of homeomorphisms $\varphi:[0,+\infty) \rightarrow[0,+\infty)$ such that both $\varphi$ and $\log \circ \varphi \circ \exp$ are convex.

(B) The class $B$ consists of the functions $\gamma: \mathbb{R} \rightarrow \mathbb{R}$ that are continuous, increasing, and convex.

For any function $\varphi$ in $A$, we define a function $\gamma$ belonging to $B$ as

$$
\gamma(t):=\log \circ \varphi \circ \exp (t)-t .
$$

Then this gives a one to one correspondence between $A$ and $B$.

Example 3.15. We cite below some specific examples of functions fulfilling the assumptions of Theorem 3.13.

(1) For a real number $p \in[1,+\infty)$, let $\varphi(s):=s^{p}$. Then the function $F_{\varphi}$ coincides with $F_{p}$ in Example 3.7 (1).

(2) Let $\varphi(s):=e^{s}-1$. Then the function $F_{\varphi}$ coincides with $F_{\exp }$ in Example 3.7 (2).

(3) Let $\varphi(s):=\sinh s$. Then $F_{\varphi}(s, t)=\operatorname{arsinh}(\sinh s+\sinh t)$.

(4) Let $\varphi(s):=s^{2}+2 s$. Then $F_{\varphi}(s, t)=\sqrt{s^{2}+2 s+t^{2}+2 t+1}-1$.

Remark 3.16. In [11], Mulholland proved that if a homeomorphism $\varphi:[0,+\infty) \rightarrow[0,+\infty)$ satisfies (3.3), then $\varphi$ is convex. In recent years, Petrík [16] gave new type examples of functions that fulfill (3.3). One of his specific examples is

$$
\varphi(s):= \begin{cases}\frac{5}{3} s & \text { if } s \in[0,1), \\ \frac{7}{3} s-\frac{2}{3} & \text { if } s \in[1,2), \\ s^{2} & \text { if } s \in[2,+\infty) .\end{cases}
$$

This $\varphi$ satisfies (3.3) but $\log \circ \varphi \circ$ exp is not convex. Of course, for this $\varphi$, the function $F_{\varphi}$ is a metric preserving function.

We show some examples of metric preserving functions that are not isotones. We say that a function $F:[0,+\infty)^{N} \rightarrow[0,+\infty)$ is tightly bounded if there exists $T>0$ such that $T \leq F\left(s_{1}, \ldots, s_{N}\right) \leq 2 T$ for every $\left(s_{i}\right)_{i=1}^{N} \in[0,+\infty)^{N} \backslash\{(0, \ldots, 0)\}$. It is easy to prove that if a function $F:[0,+\infty)^{N} \rightarrow[0,+\infty)$ is tightly bounded and $F(0, \ldots, 0)=0$, then $F$ is a metric preserving function (but is not continuous).

Theorem 3.17 ([2, Theorem 1]). Let $F$ and $G$ be two metric preserving functions on $[0,+\infty)$. Assume that there exists $r>0$ such that $F(r)=G(r)$. Define a function $H$ on $[0,+\infty)$ by

$$
H(s):= \begin{cases}F(s) & \text { if } s \in[0, r), \\ G(s) & \text { if } s \in[r,+\infty) .\end{cases}
$$

If $F$ is concave and $|G(s)-G(t)| \leq F(|s-t|)$ holds for any $s, t \in[r,+\infty)$ with $|s-t| \leq r$, then $H$ is a metric preserving function.

For example, by Theorem 3.17, the following functions are continuous metric preserving functions although these are not isotones.

$$
\begin{aligned}
& H_{1}(s):= \begin{cases}s & \text { if } s \in[0,2), \\
4-s & \text { if } s \in[2,3), \\
1 & \text { if } s \in[3,+\infty)\end{cases} \\
& H_{2}(s):= \begin{cases}s & \text { if } s \in[0,1), \\
\frac{1+s+\sin ^{2}(s-1)}{2 s} & \text { if } s \in[1,+\infty) .\end{cases}
\end{aligned}
$$

We gives counterexamples for Remark 1.2 as follows. 
Example 3.18. We define three functions $F_{n}^{1}, F_{n}^{2}$, and $F_{n}^{3}$ by

$$
\begin{aligned}
& F_{n}^{1}(s):= \begin{cases}s & \text { if } s \in[0,2), \\
4-s & \text { if } s \in\left[2,2+n^{-1}\right), \\
2-n^{-1} & \text { if } s \in\left[2+n^{-1},+\infty\right) .\end{cases} \\
& F_{n}^{2}(s):= \begin{cases}s & \text { if } s \in[0,2), \\
2 & \text { if } s \in[2, n+2), \\
s-n & \text { if } s \in[n+2, n+3), \\
n+6-s & \text { if } s \in[n+3, n+4), \\
2 & \text { if } s \in[n+4,+\infty) .\end{cases} \\
& F_{n}^{3}(s):= \begin{cases}s & \text { if } s \in[0,2), \\
2 & \text { if } s \in[2, n+2), \\
n+4-s & \text { if } s \in[n+2, n+3), \\
1 & \text { if } s \in[n+3,+\infty) .\end{cases}
\end{aligned}
$$

By Theorem 3.17, the functions $F_{n}^{i}, i=1,2,3$, belong to $\mathscr{F}^{1}$ and converge to the function $\min \{s, 2\}$ as $n \rightarrow \infty$. Let

$$
G_{n}^{i}(s, t):=F_{n}^{i}(s)+F_{n}^{i}(t)
$$

for every $i=1,2,3$. Note that $G_{n}^{i}$ belongs to $\mathscr{F}^{2}$ and converges to $\min \{s, 2\}+\min \{t, 2\}$ for every $i$. The functions $G_{n}^{1}, G_{n}^{2}$, and $G_{n}^{3}$ are counterexamples of “(ii) $\Rightarrow$ (i)”, “(iii) $\Rightarrow$ (ii)”, and “(v) $\Rightarrow$ (iv)” of Remark 1.2 respectively.

Example 3.19. The following function $F$ is a continuous metric preserving function such that $F$ is not an isotone but both $s \mapsto F(s, 0)$ and $t \mapsto F(0, t)$ are nondecreasing.

$$
F(s, t):= \begin{cases}\min \{s, 1\}+\min \{t, 1\} & \text { if } s \in[0,1) \text { or } t \in[0,1), \\ 2-\min \{s-1, t-1,1\} & \text { if } s, t \in[1,+\infty) .\end{cases}
$$

\subsection{Estimates of observable diameter}

In this subsection, we study the relation between the observable diameter and the metric preserving function. Our goal is the estimate of the observable diameter of product spaces.

Definition 3.20 (Concentration function). Let $X$ be an mm-space. We define the concentration function $\alpha_{X}$ of $X$ to be

$$
\alpha_{X}(r):=\sup _{A}\left(1-m_{X}\left(U_{r}(A)\right)\right)
$$

for $r>0$, where $A \subset X$ runs over all Borel subsets with $m_{X}(A) \geq 1 / 2$.

Proposition 3.21 ([8, Proposition 1.12], [17, Remark 2.28]).

(1) $\operatorname{ObsDiam}(X ;-\kappa) \leq 2 \inf \left\{r>0 \mid \alpha_{X}(r) \leq \kappa / 2\right\}$ for any $\kappa>0$.

(2) $\alpha_{X}(r) \leq \sup \{\kappa>0 \mid \operatorname{ObsDiam}(X ;-\kappa) \geq r\}$ for any $r>0$.

Lemma 3.22. Let $F \in \mathscr{F}^{1}$ and let $X$ be an mm-space. Denote the $m m$-space $\left(X, F \circ d_{X}, m_{X}\right)$ by $X^{F}$. Then, we have

(1) $\sup _{\varepsilon>0} \alpha_{X^{F}}(2 F(s)+\varepsilon) \leq \alpha_{X}(s)$ for any $s>0$,

(2) $\operatorname{ObsDiam}\left(X^{F} ;-2 \kappa\right) \leq 4 F(\operatorname{ObsDiam}(X ;-\kappa))$ for any $\kappa>0$.

Proof. We first prove (1). Let $\varepsilon$ and $s$ be arbitrary positive real numbers. We take any $r>0$ such that $r<$ $\alpha_{X^{F}}(2 F(s)+\varepsilon)$. There exists a Borel subset $A \subset X$ such that $m_{X}(A) \geq 1 / 2$ and

$$
1-m_{X}\left(U_{2 F(s)+\varepsilon}^{F}(A)\right)>r,
$$


where $U_{r}^{F}(A)$ is the $r$-open neighborhood of $A$ with respect to $F \circ d_{X}$. By Corollary 3.3 (2), we have $U_{S}(A) \subset$ $U_{2 F(s)+\varepsilon}^{F}(A)$. Indeed, for $x \in X$, if there exists $x^{\prime} \in A$ such that $d_{X}\left(x, x^{\prime}\right)<s$, then $F\left(d_{X}\left(x, x^{\prime}\right)\right) \leq 2 F(s)$ by Corollary 3.3 (2). We obtain

$$
\inf _{x^{\prime} \in A} F\left(d_{X}\left(x, x^{\prime}\right)\right)<2 F(s)+\varepsilon,
$$

which implies $U_{s}(A) \subset U_{2 F(s)+\varepsilon}^{F}(A)$. By this, we have

$$
r<1-m_{X}\left(U_{2 F(s)+\varepsilon}^{F}(A)\right) \leq 1-m_{X}\left(U_{s}(A)\right) \leq \alpha_{X}(s) .
$$

Since $r$ is arbitrary, we obtain $\alpha_{X^{F}}(2 F(s)+\varepsilon) \leq \alpha_{X}(s)$.

We next prove (2). Let $\varepsilon$ and $\kappa$ be arbitrary positive real numbers. Setting

$$
s_{\varepsilon}:=\operatorname{Obs} \operatorname{Diam}(X ;-\kappa)+\varepsilon,
$$

by (1) of this lemma and Proposition 3.21 (2), we have

$$
\alpha_{X^{F}}\left(2 F\left(s_{\varepsilon}\right)+\varepsilon\right) \leq \alpha_{X}\left(s_{\varepsilon}\right) \leq \kappa .
$$

Combining this and Proposition 3.21 (1) implies

$$
\operatorname{ObsDiam}\left(X^{F} ;-2 \kappa\right) \leq 4 F\left(s_{\varepsilon}\right)+2 \varepsilon .
$$

As $\varepsilon \rightarrow 0$, we obtain $\operatorname{Obs} \operatorname{Diam}\left(X^{F} ;-2 \kappa\right) \leq 4 F(\operatorname{ObsDiam}(X ;-\kappa))$. The proof is completed.

Lemma 3.23 ([17, in the proof of Proposition 7.32]). Let $X$ and $Y$ be two mm-spaces and let $p \in[1,+\infty]$. Given $f \in \mathcal{L i p}_{1}\left(X \times_{p} Y\right)$, we define functions $g: X \rightarrow \mathbb{R}$ and $h: Y \rightarrow \mathbb{R}$ by

$$
g(x):=\operatorname{lm}\left(f(x, \cdot) ; m_{Y}\right) \text { and } h(y):=\operatorname{lm}\left(f(\cdot, y) ; m_{X}\right)
$$

for $x \in X$ and $y \in Y$. Then we have $g \in \mathcal{L i p}_{1}(X)$ and $h \in \mathcal{L i p}_{1}(Y)$.

Proposition 3.24. Let $X$ and $Y$ be two $m m$-spaces and let $F, G \in \mathscr{F}^{2}$. If $F \leq G$, that is, $F(s, t) \leq G(s, t)$ for every $s, t$, then we have

$$
\operatorname{ObsDiam}\left(X \times_{F} Y ;-\kappa\right) \leq \operatorname{ObsDiam}\left(X \times_{G} Y ;-\kappa\right)
$$

for any $\kappa>0$.

Proof. We take any $f \in \mathcal{L i p}{ }_{1}\left(X \times_{F} Y\right)$. Since

$$
\left|f(x, y)-f\left(x^{\prime}, y^{\prime}\right)\right| \leq F\left(d_{X}\left(x, x^{\prime}\right), d_{Y}\left(y, y^{\prime}\right)\right) \leq G\left(d_{X}\left(x, x^{\prime}\right), d_{Y}\left(y, y^{\prime}\right)\right),
$$

we have $f \in \mathcal{L} \operatorname{Lip}_{1}\left(X \times_{G} Y\right)$. Thus

$$
\operatorname{diam}\left(f_{\star}\left(m_{X} \otimes m_{Y}\right) ; 1-\kappa\right) \leq \operatorname{ObsDiam}\left(X \times_{G} Y ;-\kappa\right),
$$

which implies (3.9). The proof is completed.

Remark 3.25. It is well-known that the observable diameter is monotone with respect to the Lipschitz order which is a partial order relation over $X$ (see [17, Proposition 2.18]). Proposition 3.24 is a special case of this.

The following lemmas are keys to the proof of Theorem 1.1.

Lemma 3.26. Let $X$ and $Y$ be two $m m$-spaces and let $p \in[1,+\infty]$. Then we have

$$
\operatorname{ObsDiam}\left(X \times_{p} Y ;-\left(\kappa+\kappa^{\prime}\right)\right) \leq \operatorname{ObsDiam}(X ;-\kappa)+2 \operatorname{Obs} \operatorname{Diam}\left(Y ;-\kappa^{\prime}\right)
$$

for any $\kappa>0$ and any $\kappa^{\prime} \in(0,1 / 2)$. 
Lemma 3.27. Let $X$ and $Y$ be two $m m$-spaces and let $F \in \mathscr{F}^{2}$. Then we have

$$
\operatorname{ObsDiam}\left(X \times_{F} Y ;-2\left(\kappa+\kappa^{\prime}\right)\right) \leq 4 F(\operatorname{ObsDiam}(X ;-\kappa), 0)+8 F\left(0, \operatorname{ObsDiam}\left(Y ;-\kappa^{\prime}\right)\right)
$$

for any $\kappa>0$ and any $\kappa^{\prime} \in(0,1 / 4)$.

Proof of Lemma 3.26. We take any real numbers $\varepsilon, \delta>0$ with

$$
\operatorname{ObsDiam}(X ;-\kappa)<\varepsilon \text { and } \operatorname{ObsDiam}\left(Y ;-\kappa^{\prime}\right)<\delta,
$$

and take any $f \in \mathcal{L i p}_{1}\left(X \times_{p} Y\right)$. It suffices to prove

$$
\operatorname{diam}\left(f_{\star}\left(m_{X} \otimes m_{Y}\right) ; 1-\left(\kappa+\kappa^{\prime}\right)\right) \leq \varepsilon+2 \delta .
$$

For all $x \in X$, let $f_{x}:=f(x, \cdot) \in \mathcal{L i p}_{1}(Y)$. We define $g: X \rightarrow \mathbb{R}$ by

$$
g(x):=\operatorname{lm}\left(f_{x} ; m_{Y}\right)
$$

for $x \in X$. By Lemma 3.23, we have $g \in \mathcal{L} \operatorname{Lip}_{1}(X)$. By $\operatorname{ObsDiam}(X ;-\kappa)<\varepsilon$, we have $\operatorname{diam}\left(g_{\star} m_{X} ; 1-\kappa\right)<\varepsilon$. Thus there exists an interval $I \subset \mathbb{R}$ such that

$$
g_{\star} m_{X}(I) \geq 1-\kappa \quad \text { and } \operatorname{diam} I<\varepsilon .
$$

We take countable dense points $\left\{x_{i}\right\}_{i \in \mathbb{N}}$ in $g^{-1}(I)$. By ObsDiam $\left(Y ;-\kappa^{\prime}\right)<\delta$, we have $\operatorname{diam}\left(\left(f_{x_{i}}\right) \star m_{Y} ; 1-\kappa^{\prime}\right)<\delta$ for any $i \in \mathbb{N}$. For each $i \in \mathbb{N}$, there exists an interval $J_{i} \subset \mathbb{R}$ such that

$$
\left(f_{x_{i}}\right) \star m_{Y}\left(J_{i}\right) \geq 1-\kappa^{\prime} \quad \text { and } \operatorname{diam} J_{i}<\delta .
$$

Combining $\left(f_{x_{i}}\right)_{\star} m_{Y}\left(J_{i}\right)>1 / 2$ and the connectedness of $J_{i}$ leads to $\operatorname{lm}\left(f_{x_{i}} ; m_{Y}\right) \in J_{i}$.

We take any $\eta>0$ and fix it. We define a Borel set $\tilde{A}_{\eta}$ by

$$
\tilde{A}_{\eta}:=\bigsqcup_{i \in \mathbb{N}}\left(U_{\eta}^{i} \times f_{x_{i}}^{-1}\left(J_{i}\right)\right) \subset X \times Y,
$$

where $U_{\eta}^{1}:=U_{\eta}\left(x_{1}\right)$ and $U_{\eta}^{i}:=U_{\eta}\left(x_{i}\right) \backslash \bigcup_{j<i} U_{\eta}^{j}$ for $i \geq 2$. Then we see that

$$
\begin{aligned}
m_{X} \otimes m_{Y}\left(\tilde{A}_{\eta}\right) & =\sum_{i=1}^{\infty} m_{X}\left(U_{\eta}^{i}\right) m_{Y}\left(f_{x_{i}}^{-1}\left(J_{i}\right)\right) \geq\left(1-\kappa^{\prime}\right) \sum_{i=1}^{\infty} m_{X}\left(U_{\eta}^{i}\right) \\
& \geq\left(1-\kappa^{\prime}\right) m_{X}\left(g^{-1}(I)\right) \geq(1-\kappa)\left(1-\kappa^{\prime}\right) \geq 1-\left(\kappa+\kappa^{\prime}\right) .
\end{aligned}
$$

A closed subset $A_{\eta}$ of $\mathbb{R}$ is defined as the closure of $f\left(\tilde{A}_{\eta}\right)$. Then we have

$$
f_{\star}\left(m_{X} \otimes m_{Y}\right)\left(A_{\eta}\right) \geq m_{X} \otimes m_{Y}\left(\tilde{A}_{\eta}\right) \geq 1-\left(\kappa+\kappa^{\prime}\right) .
$$

Moreover, we have

$$
\operatorname{diam} A_{\eta}=\operatorname{diam} f\left(\tilde{A}_{\eta}\right) \leq \varepsilon+2 \delta+2 \eta .
$$

Indeed, for any $(x, y) \in U_{\eta}^{i} \times f_{x_{i}}^{-1}\left(J_{i}\right)$ and $\left(x^{\prime}, y^{\prime}\right) \in U_{\eta}^{j} \times f_{x_{j}}^{-1}\left(U_{j}\right)$,

$$
\begin{aligned}
& \left|f(x, y)-f\left(x^{\prime}, y^{\prime}\right)\right| \\
\leq & \left|f(x, y)-f\left(x_{i}, y\right)\right|+\left|f\left(x_{i}, y\right)-g\left(x_{i}\right)\right|+\left|g\left(x_{i}\right)-g\left(x_{j}\right)\right| \\
& +\left|g\left(x_{j}\right)-f\left(x_{j}, y^{\prime}\right)\right|+\left|f\left(x_{j}, y^{\prime}\right)-f\left(x^{\prime}, y^{\prime}\right)\right| \\
\leq & d_{X}\left(x, x_{i}\right)+\operatorname{diam} J_{i}+\operatorname{diam} I+\operatorname{diam} J_{j}+d_{X}\left(x_{j}, x^{\prime}\right) \\
\leq & \varepsilon+2 \delta+2 \eta .
\end{aligned}
$$

Thus we have $\operatorname{diam}\left(f_{\star}\left(m_{X} \otimes m_{Y}\right) ; 1-\left(\kappa+\kappa^{\prime}\right)\right) \leq \operatorname{diam} A_{\eta} \leq \varepsilon+2 \delta+2 \eta$, which implies (3.12). This completes the proof. 
Proof of Lemma 3.27. Let $\kappa$ and $\kappa^{\prime}$ be two real numbers with $\kappa>0$ and $\kappa^{\prime} \in(0,1 / 4)$ and let $F \in \mathscr{F}^{2}$. The two functions $F_{1}$ and $F_{2}$ on $[0,+\infty)$ defined by

$$
F_{1}(s):=F(s, 0), \quad F_{2}(t):=F(0, t)
$$

for $s, t \in[0,+\infty)$ belong to $\mathscr{F}^{1}$, and then the function $G$ on $[0,+\infty)^{2}$ defined by

$$
G(s, t):=F_{1}(s)+F_{2}(t)=F(s, 0)+F(0, t)
$$

for $s, t \in[0,+\infty)$ belongs to $\mathscr{F}^{2}$. Since $F \leq G$ holds, by Proposition 3.24, we have

$$
\operatorname{Obs} \operatorname{Diam}\left(X \times_{F} Y ;-2\left(\kappa+\kappa^{\prime}\right)\right) \leq \operatorname{Obs} \operatorname{Diam}\left(X \times_{G} Y ;-2\left(\kappa+\kappa^{\prime}\right)\right) .
$$

Taking into account that $X \times_{G} Y$ is mm-isomorphic to

$$
\left(X, F_{1} \circ d_{X}, m_{X}\right) \times_{1}\left(Y, F_{2} \circ d_{Y}, m_{Y}\right),
$$

by Lemma 3.26 and Lemma 3.22 (2), we have

$$
\begin{aligned}
& \operatorname{ObsDiam}\left(X \times_{G} Y ;-2\left(\kappa+\kappa^{\prime}\right)\right) \\
& \leq 4 F_{1}(\operatorname{Obs} \operatorname{Diam}(X ;-\kappa))+8 F_{2}\left(\operatorname{Obs} \operatorname{Diam}\left(Y ;-\kappa^{\prime}\right)\right) \\
& =4 F(\operatorname{ObsDiam}(X ;-\kappa), 0)+8 F\left(0, \operatorname{ObsDiam}\left(Y ;-\kappa^{\prime}\right)\right) .
\end{aligned}
$$

This completes the proof.

We use the following example in Section 5.

Example 3.28. Let $S^{n}\left(r_{n}\right), n=1,2, \ldots$, be the sphere of radius $r_{n}>0$ in $\mathbb{R}^{n+1}$ and let $\sigma^{n}$ be the Riemannian volume measure on $S^{n}\left(r_{n}\right)$ normalized as $\sigma^{n}\left(S^{n}\left(r_{n}\right)\right)=1$. Let $F_{n}$ be the function in $\mathscr{F}^{1}$ defined by

$$
F_{n}(s):= \begin{cases}2 r_{n} \sin \frac{s}{2 r_{n}} & \text { if } s \leq \pi r_{n}, \\ 2 r_{n} & \text { if } s>\pi r_{n}\end{cases}
$$

for $s \in[0,+\infty)$. Let $d$ be the Riemannian distance on $S^{n}\left(r_{n}\right)$ and let $\|\cdot\|$ be the Euclidean norm on $\mathbb{R}^{n+1}$. We see that

$$
\left\|x-x^{\prime}\right\|=F_{n}\left(d\left(x, x^{\prime}\right)\right) \leq d\left(x, x^{\prime}\right)
$$

for any $x, x^{\prime} \in S^{n}\left(r_{n}\right)$. Thus, by Proposition 3.24, we have

$$
\operatorname{ObsDiam}\left(\left(S^{n}\left(r_{n}\right),\|\cdot\|, \sigma^{n}\right) ;-\kappa\right) \leq \operatorname{ObsDiam}\left(\left(S^{n}\left(r_{n}\right), d, \sigma^{n}\right) ;-\kappa\right)
$$

for any $\kappa>0$. Combining this and Example 2.16 implies that the sequence $\left\{\left(S^{n}\left(r_{n}\right),\|\cdot\|, \sigma^{n}\right)\right\}_{n \in \mathbb{N}}$ is a Lévy family if $r_{n}=o(\sqrt{n})$.

\section{Convergence of product spaces}

\subsection{Box-convergence of product spaces}

The purpose of this subsection is to prove the following two propositions and their corollaries. We denote by $\mathbb{N}$ the set of positive integers and by $\mathscr{F}^{N}$ the set of continuous metric preserving functions on $[0,+\infty)^{N}$.

Proposition 4.1. Let $X, Y, Z$, and $W$ be four $m m$-spaces and let $F \in \mathscr{F}^{2}$. Then we have

$$
\square\left(X \times_{F} Z, Y \times_{F} W\right) \leq \max \left\{\square(X, Y)+\square(Z, W), 2 F\left(\frac{1}{2} \square(X, Y), \frac{1}{2} \square(Z, W)\right)\right\} .
$$

In particular, for any $p \in[1,+\infty]$, it holds that

$$
\square\left(X \times_{p} Z, Y \times_{p} W\right) \leq \square(X, Y)+\square(Z, W) .
$$


Proposition 4.2. Let $\left\{X_{n}\right\}_{n \in \mathbb{N}}$ and $\left\{Y_{n}\right\}_{n \in \mathbb{N}}$ be two sequences of mm-spaces $\square$-converging to mm-spaces $X$ and $Y$ respectively. Let $F_{n}, n=1,2, \ldots$, and $F$ be functions in $\mathscr{F}^{2}$ such that $F_{n}$ converges pointwise to $F$ as $n \rightarrow \infty$. Then the sequence $\left\{X_{n} \times_{F_{n}} Y_{n}\right\}_{n \in \mathbb{N}}$ of their product spaces $\square$-converges to the product space $X \times_{F} Y$.

Corollary 4.3. Let $\left\{X_{n}\right\}_{n \in \mathbb{N}}$ and $\left\{Y_{n}\right\}_{n \in \mathbb{N}}$ be two sequences of mm-spaces $\square$-converging to mm-spaces $X$ and $Y$ respectively. Assume that $p_{n} \in[1,+\infty]$ converges to $p \in[1,+\infty]$ as $n \rightarrow \infty$. Then the sequence $\left\{X_{n} \times_{p_{n}} Y_{n}\right\}_{n \in \mathbb{N}}$ of their $l_{p_{n}}$-product spaces $\square$-converges to the $l_{p}$-product space $X \times_{p} Y$.

Corollary 4.4. Let $\left\{X_{n}\right\}_{n \in \mathbb{N}}$ be a sequence of mm-spaces $\square$-converging to an mm-space $X$. Let $F_{n}, n=1,2, \ldots$, and $F$ be functions in $\mathscr{F}^{1}$ such that $F_{n}$ converges pointwise to $F$ as $n \rightarrow \infty$. Then the sequence $\left\{\left(X_{n}, F_{n} \circ\right.\right.$ $\left.\left.d_{X_{n}}, m_{X_{n}}\right)\right\}_{n \in \mathbb{N}}$ of $m m$-spaces $\square$-converges to the $m m$-space $\left(X, F \circ d_{X}, m_{X}\right)$.

Given two maps $f: X \rightarrow X^{\prime}$ and $g: Y \rightarrow Y^{\prime}$, a map $f \times g: X \times Y \rightarrow X^{\prime} \times Y^{\prime}$ is defined by

$$
(f \times g)(x, y):=(f(x), g(y))
$$

for $(x, y) \in X \times Y$. In this paper, for two maps $f$ and $g$, we always use the notation $f \times g$ in the sense of (4.3).

Proof of Proposition 4.1. This proposition is a generalization of [13, Lemma 3.3] and its proof is similar to that of [13, Lemma 3.3].

Take any two real numbers $\varepsilon, \delta>0$ such that $\square(X, Y)<\varepsilon, \square(Z, W)<\delta$. Then there exist four parameters $\varphi: I \rightarrow X, \psi: I \rightarrow Y, \xi: I \rightarrow Z$, and $\eta: I \rightarrow W$ of every $X, Y, Z$, and $W$ respectively, and two Borel subsets $I_{1}$, $I_{2} \subset I$ such that

$$
\begin{aligned}
& \left|d_{X}(\varphi(s), \varphi(t))-d_{Y}(\psi(s), \psi(t))\right|<\varepsilon \text { for any } s, t \in I_{1}, \\
& \left|d_{Z}(\xi(s), \xi(t))-d_{W}(\eta(s), \eta(t))\right|<\delta \text { for any } s, t \in I_{2}, \\
& \mathcal{L}^{1}\left(I_{1}\right) \geq 1-\varepsilon, \quad \text { and } \quad \mathcal{L}^{1}\left(I_{2}\right) \geq 1-\delta .
\end{aligned}
$$

Let $\Phi$ be a parameter of $\left(I \times I, \mathcal{L}^{1} \otimes \mathcal{L}^{1}\right)$, i.e., a Borel measurable map $\Phi: I \rightarrow I \times I$ with

$$
\Phi_{\star} \mathcal{L}^{1}=\mathcal{L}^{1} \otimes \mathcal{L}^{1}
$$

where the existence of $\Phi$ follows from Lemma 2.8. Then the Borel measurable map $(\varphi \times \xi) \circ \Phi: I \rightarrow X \times Z$ is a parameter of $X \times Z$. Indeed, we have

$$
((\varphi \times \xi) \circ \Phi)_{\star} \mathcal{L}^{1}=(\varphi \times \xi) \star\left(\mathcal{L}^{1} \otimes \mathcal{L}^{1}\right)=\varphi \star \mathcal{L}^{1} \otimes \xi \star \mathcal{L}^{1}=m_{X} \otimes m_{Z}
$$

Similarly, the Borel measurable map $(\psi \times \eta) \circ \Phi: I \rightarrow Y \times W$ is a parameter of $Y \times W$. Setting $I_{0}:=\Phi^{-1}\left(I_{1} \times I_{2}\right)$, we have

$$
\mathcal{L}^{1}\left(I_{0}\right)=\mathcal{L}^{1}\left(\Phi^{-1}\left(I_{1} \times I_{2}\right)\right)=\mathcal{L}^{1}\left(I_{1}\right) \mathcal{L}^{1}\left(I_{2}\right) \geq(1-\varepsilon)(1-\delta) \geq 1-(\varepsilon+\delta) .
$$

In addition, we define four functions $\varphi^{\prime}, \psi^{\prime}, \xi^{\prime}, \eta^{\prime}$ by

$$
\left(\varphi^{\prime}(s), \xi^{\prime}(s)\right)=(\varphi \times \xi) \circ \Phi(s) \text { and }\left(\psi^{\prime}(s), \eta^{\prime}(s)\right)=(\psi \times \eta) \circ \Phi(s)
$$

for any $s \in I$. Then, for any $s, t \in I_{0}$, we have

$$
\begin{aligned}
& \left|d_{F}((\varphi \times \xi) \circ \Phi(s),(\varphi \times \xi) \circ \Phi(t))-d_{F}((\psi \times \eta) \circ \Phi(s),(\psi \times \eta) \circ \Phi(t))\right| \\
& =\left|F\left(d_{X}\left(\varphi^{\prime}(s), \varphi^{\prime}(t)\right), d_{Z}\left(\xi^{\prime}(s), \xi^{\prime}(t)\right)\right)-F\left(d_{Y}\left(\psi^{\prime}(s), \psi^{\prime}(t)\right), d_{W}\left(\eta^{\prime}(s), \eta^{\prime}(t)\right)\right)\right| \\
& \leq F\left(\left|d_{X}\left(\varphi^{\prime}(s), \varphi^{\prime}(t)\right)-d_{Y}\left(\psi^{\prime}(s), \psi^{\prime}(t)\right)\right|,\left|d_{Z}\left(\xi^{\prime}(s), \xi^{\prime}(t)\right)-d_{W}\left(\eta^{\prime}(s), \eta^{\prime}(t)\right)\right|\right) \\
& \leq 2 F\left(\frac{1}{2} \varepsilon, \frac{1}{2} \delta\right),
\end{aligned}
$$

where the inequalities follow from Corollary 3.3. Thus we have

$$
\square\left(X \times_{F} Z, Y \times_{F} W\right) \leq \max \left\{\varepsilon+\delta, 2 F\left(\frac{1}{2} \varepsilon, \frac{1}{2} \delta\right)\right\},
$$


so that we obtain (4.1). Furthermore, in the case of $F=F_{p}$ in Example 3.7 (1) for $p \in[1,+\infty]$, we see that $F_{p}(s, t) \leq s+t$ for every $s, t$. Combining this and (4.4) implies

$$
\square\left(X \times_{F} Z, Y \times_{F} W\right) \leq \varepsilon+\delta,
$$

which gives (4.2). The proof of the proposition is completed.

We need the following two lemmas for the proof of Proposition 4.2.

Lemma 4.5. Let $\mu, \mu^{\prime}$ be two Borel probability measures on a separable metric space $X$ and let $v, v^{\prime}$ be two Borel probability measures on a separable metric space $Y$. Let $F$ be a function in $\mathscr{F}^{2}$. Then we have

$$
d_{\mathrm{P}}^{(\lambda)}\left(\mu \otimes v, \mu^{\prime} \otimes v^{\prime}\right) \leq \max \left\{d_{\mathrm{P}}^{(\lambda)}\left(\mu, \mu^{\prime}\right)+d_{\mathrm{P}}^{(\lambda)}\left(v, v^{\prime}\right), 2 F\left(d_{\mathrm{P}}^{(\lambda)}\left(\mu, \mu^{\prime}\right), d_{\mathrm{P}}^{(\lambda)}\left(v, v^{\prime}\right)\right)\right\}
$$

for any $\lambda>0$, where $d_{\mathrm{P}}^{(\lambda)}$ in the left-hand side is with respect to $d_{F}$.

Proof. We take any two real numbers $\varepsilon$ and $\delta$ such that $d_{\mathrm{P}}^{(\lambda)}\left(\mu, \mu^{\prime}\right)<\varepsilon, d_{\mathrm{P}}^{(\lambda)}\left(v, v^{\prime}\right)<\delta$ and fix them. For the proof of (4.5), it suffices to prove that

$$
(\mu \otimes v)(A) \leq\left(\mu^{\prime} \otimes v^{\prime}\right)\left(U_{2 F(\varepsilon, \delta)+\eta}(A)\right)+\lambda(\varepsilon+\delta)
$$

for any Borel subset $A \subset X \times Y$ and for any $\eta>0$. The famous $\pi$ - $\lambda$ theorem shows that if we prove

$$
(\mu \otimes v)(B \times C) \leq\left(\mu^{\prime} \otimes v^{\prime}\right)\left(U_{2 F(\varepsilon, \delta)+\eta}(B \times C)\right)+\lambda(\varepsilon+\delta)
$$

for any Borel subsets $B \subset X$ and $C \subset Y$, then we obtain (4.6) for any Borel subset $A \subset X \times Y$. Let us prove (4.7). By $d_{\mathrm{P}}^{(\lambda)}\left(\mu, \mu^{\prime}\right)<\varepsilon$ and $d_{\mathrm{P}}^{(\lambda)}\left(\nu, v^{\prime}\right)<\delta$, we have

$$
\begin{aligned}
& (\mu \otimes v)(B \times C)(=\mu(B) v(C)) \\
& \leq\left(\mu^{\prime}\left(U_{\varepsilon}(B)\right)+\lambda \varepsilon\right) v(C) \\
& \leq \mu^{\prime}\left(U_{\varepsilon}(B)\right) v(C)+\lambda \varepsilon \\
& \leq \mu^{\prime}\left(U_{\varepsilon}(B)\right)\left(v^{\prime}\left(U_{\delta}(C)\right)+\lambda \delta\right)+\lambda \varepsilon \\
& \leq \mu^{\prime}\left(U_{\varepsilon}(B)\right) v^{\prime}\left(U_{\delta}(C)\right)+\lambda(\varepsilon+\delta) \\
& \leq\left(\mu^{\prime} \otimes v^{\prime}\right)\left(U_{2 F(\varepsilon, \delta)+\eta}(B \times C)\right)+\lambda(\varepsilon+\delta)
\end{aligned}
$$

for any Borel subsets $B \subset X$ and $C \subset Y$, where the last inequality follows from Corollary 3.3 (2). Thus we obtain (4.7) and then (4.5). The proof is completed.

Lemma 4.6. Let $F_{n}, n=1,2, \ldots$, and $F$ be metric preserving functions. If $F$ is continuous and $F_{n}$ converges pointwise to $F$, then $F_{n}$ uniformly converges to $F$ on compact sets.

Proof. We take any compact set $K \subset[0,+\infty)^{2}$ and any real number $\varepsilon>0$. Let us prove that

$$
\sup _{(s, t) \in K}\left|F_{n}(s, t)-F(s, t)\right| \leq 7 \varepsilon
$$

holds for every sufficiently large $n$. By the continuity of $F$, there exists a real number $\delta>0$ such that $F(\delta, \delta) \leq \varepsilon$. By the compactness of $K$, we find finitely many points $\left\{\left(s_{i}, t_{i}\right)\right\}_{i=1}^{k}$ in $K$ such that

$$
K \subset \bigcup_{i=1}^{k}\left(U_{\delta}\left(s_{i}\right) \times U_{\delta}\left(t_{i}\right)\right) .
$$

Let $N \in \mathbb{N}$ be a number such that

$$
\max _{i=1, \ldots, k}\left|F_{n}\left(s_{i}, t_{i}\right)-F\left(s_{i}, t_{i}\right)\right| \leq \varepsilon \text { and }\left|F_{n}(\delta, \delta)-F(\delta, \delta)\right| \leq \varepsilon
$$


hold for all $n \geq N$. Given a fixed point $(s, t) \in K$, we find $i \in\{1, \ldots, k\}$ such that $(s, t) \in U_{\delta}\left(s_{i}\right) \times U_{\delta}\left(t_{i}\right)$. By Corollary 3.3, we have

$$
\begin{aligned}
& \left|F_{n}(s, t)-F(s, t)\right| \\
& \leq\left|F_{n}\left(s_{i}, t_{i}\right)-F\left(s_{i}, t_{i}\right)\right|+F_{n}\left(\left|s-s_{i}\right|,\left|t-t_{i}\right|\right)+F\left(\left|s-s_{i}\right|,\left|t-t_{i}\right|\right) \\
& \leq\left|F_{n}\left(s_{i}, t_{i}\right)-F\left(s_{i}, t_{i}\right)\right|+2 F_{n}(\delta, \delta)+2 F(\delta, \delta) \\
& \leq\left|F_{n}\left(s_{i}, t_{i}\right)-F\left(s_{i}, t_{i}\right)\right|+2\left|F_{n}(\delta, \delta)-F(\delta, \delta)\right|+4 F(\delta, \delta) \\
& \leq 7 \varepsilon
\end{aligned}
$$

for every $n \geq N$. Thus we obtain (4.8). This completes the proof.

Proof of Proposition 4.2. It is sufficient to prove that, for any real number $\varepsilon>0$, there exists $N(\varepsilon) \in \mathbb{N}$ such that an $\varepsilon$-mm-isomorphism $\Phi_{n}: X_{n} \times_{F_{n}} Y_{n} \rightarrow X \times_{F} Y$ exists for each $n \geq N(\varepsilon)$. Take any $\varepsilon>0$ and fix it. By the inner regularity of probability measures $m_{X}$ and $m_{Y}$, there exist compact sets $K \subset X$ and $K^{\prime} \subset Y$ such that

$$
m_{X}(K) \geq 1-\varepsilon \quad \text { and } \quad m_{Y}\left(K^{\prime}\right) \geq 1-\varepsilon .
$$

Let $D_{\varepsilon}:=\max \left\{\operatorname{diam} K\right.$, diam $\left.K^{\prime}\right\}+3 \varepsilon$. Since $\left\{X_{n}\right\}_{n \in \mathbb{N}},\left\{Y_{n}\right\}_{n \in \mathbb{N}} \square$-converge to $X, Y$ respectively and $F_{n}$ uniformly converges to $F$ on $\left[0, D_{\varepsilon}\right]^{2}$ following from Lemma 4.6, there exists $N(\varepsilon) \in \mathbb{N}$ such that, for any $n \geq N(\varepsilon)$,

- an $\varepsilon$-mm-isomorphism $f_{n}: X_{n} \rightarrow X$ exists,

- an $\varepsilon$-mm-isomorphism $g_{n}: Y_{n} \rightarrow Y$ exists,

- $\left|F_{n}(s, t)-F(s, t)\right|<\varepsilon$ for all $s, t \in\left[0, D_{\varepsilon}\right]$.

If we prove that the map $f_{n} \times g_{n}$ is a $(4 F(\varepsilon, \varepsilon)+6 \varepsilon)$-mm-isomorphism for any $n \geq N(\varepsilon)$, then we obtain the conclusion with $\Phi_{n}=f_{n} \times g_{n}$. Take any $n \geq N(\varepsilon)$ and fix it. Let us prove that the map $f_{n} \times g_{n}$ is a $(4 F(\varepsilon, \varepsilon)+6 \varepsilon)$ $\mathrm{mm}$-isomorphism. By Lemma 4.5, taking

$$
\left(f_{n} \times g_{n}\right) \star\left(m_{X_{n}} \otimes m_{Y_{n}}\right)=f_{n \star} m_{X_{n}} \otimes g_{n *} m_{Y_{n}}
$$

into account, we have

$$
d_{\mathrm{P}}\left(\left(f_{n} \times g_{n}\right) \star\left(m_{X_{n}} \otimes m_{Y_{n}}\right), m_{X} \otimes m_{Y}\right) \leq \max \{2 \varepsilon, 4 F(\varepsilon, \varepsilon)\} .
$$

Let $X_{n}^{\prime}, Y_{n}^{\prime}$ be nonexceptional domains of $f_{n}, g_{n}$ respectively and let

$$
\widetilde{X}_{n}:=X_{n}^{\prime} \cap f_{n}^{-1}\left(U_{\varepsilon}(K)\right) \quad \text { and } \quad \widetilde{Y}_{n}:=Y_{n}^{\prime} \cap g_{n}^{-1}\left(U_{\varepsilon}\left(K^{\prime}\right)\right) .
$$

Since $d_{\mathrm{P}}\left(f_{n \star} m_{X_{n}}, m_{X}\right) \leq \varepsilon$, we have

$$
m_{X_{n}}\left(\widetilde{X}_{n}\right) \geq m_{X_{n}}\left(X_{n}^{\prime}\right)+m_{X_{n}}\left(f_{n}^{-1}\left(U_{\varepsilon}(K)\right)\right)-1 \geq m_{X_{n}}\left(X_{n}^{\prime}\right)+\left(m_{X}(K)-\varepsilon\right)-1 \geq 1-3 \varepsilon .
$$

Similarly, $d_{\mathrm{P}}\left(g_{n \star} m_{Y_{n}}, m_{Y}\right) \leq \varepsilon$ implies $m_{Y_{n}}\left(\widetilde{Y}_{n}\right) \geq 1-3 \varepsilon$. Thus we obtain

$$
m_{X_{n}} \otimes m_{Y_{n}}\left(\widetilde{X}_{n} \times \widetilde{Y}_{n}\right) \geq 1-6 \varepsilon .
$$

Furthermore we see that $\operatorname{diam} \widetilde{X}_{n} \leq D_{\varepsilon}$ and $\operatorname{diam} \widetilde{Y}_{n} \leq D_{\varepsilon}$. Indeed,

$$
d_{X_{n}}\left(x, x^{\prime}\right) \leq d_{X}\left(f_{n}(x), f_{n}\left(x^{\prime}\right)\right)+\varepsilon \leq \operatorname{diam} K+3 \varepsilon \leq D_{\varepsilon}
$$

for any $x, x^{\prime} \in \widetilde{X}_{n}$. One can check diam $\widetilde{Y}_{n} \leq D_{\varepsilon}$ similarly. Therefore, for any $(x, y),\left(x^{\prime}, y^{\prime}\right) \in \widetilde{X}_{n} \times \widetilde{Y}_{n}$, we have

$$
\begin{aligned}
& \left|d_{F_{n}}\left((x, y),\left(x^{\prime}, y^{\prime}\right)\right)-d_{F}\left(\left(f_{n} \times g_{n}\right)(x, y),\left(f_{n} \times g_{n}\right)\left(x^{\prime}, y^{\prime}\right)\right)\right| \\
= & \left|F_{n}\left(d_{X_{n}}\left(x, x^{\prime}\right), d_{Y_{n}}\left(y, y^{\prime}\right)\right)-F\left(d_{X}\left(f_{n}(x), f_{n}\left(x^{\prime}\right)\right), d_{Y}\left(g_{n}(y), g_{n}\left(y^{\prime}\right)\right)\right)\right| \\
\leq & \left|F_{n}\left(d_{X_{n}}\left(x, x^{\prime}\right), d_{Y_{n}}\left(y, y^{\prime}\right)\right)-F\left(d_{X_{n}}\left(x, x^{\prime}\right), d_{Y_{n}}\left(y, y^{\prime}\right)\right)\right| \\
& +F\left(\left|d_{X_{n}}\left(x, x^{\prime}\right)-d_{X}\left(f_{n}(x), f_{n}\left(x^{\prime}\right)\right)\right|,\left|d_{Y_{n}}\left(y, y^{\prime}\right)-d_{Y}\left(g_{n}(y), g_{n}\left(y^{\prime}\right)\right)\right|\right) \\
\leq & \varepsilon+2 F(\varepsilon, \varepsilon) .
\end{aligned}
$$

Combining this with (4.9) and (4.10) means that the map $f_{n} \times g_{n}$ is a $(4 F(\varepsilon, \varepsilon)+6 \varepsilon)$-mm-isomorphism. The proof of the proposition is completed. 
Proof of Corollary 4.3. We apply Proposition 4.2 with $F_{n}=F_{p_{n}}, n=1,2, \ldots$, and $F=F_{p}$, where $F_{p}$ is the function of Example 3.7 (1).

Proof of Corollary 4.4. Let $Y_{n}, n=1,2, \ldots$, and $Y$ be one-point mm-spaces and let $G_{n}$ and $G$ be the functions on $[0,+\infty)^{2}$ defined by

$$
G_{n}(s, t):=F_{n}(s)+t \quad \text { and } \quad G(s, t):=F(s)+t
$$

for $s, t \in[0,+\infty)$. We just apply Proposition 4.2. Note that the mm-space $X_{n} \times_{G_{n}} Y_{n}$ is mm-isomorphic to $\left(X_{n}, F_{n} \circ d_{X_{n}}, m_{X_{n}}\right)$.

\subsection{Concentration of product spaces}

Our goals in this subsection are to prove half of Theorem 1.1, and to obtain Corollary 1.3 and half of Theorem 1.4 as its corollaries.

Definition 4.7 (1-Lipschitz up to an additive error). Let $X$ be an mm-space and $Y$ be a metric space. A map $f: X \rightarrow Y$ is said to be 1-Lipschitz up to (an additive error) $\varepsilon \geq 0$ if there exists a Borel subset $X_{0} \subset X$ such that

(1) $m_{X}\left(X_{0}\right) \geq 1-\varepsilon$,

(2) $d_{Y}\left(f(x), f\left(x^{\prime}\right)\right) \leq d_{X}\left(x, x^{\prime}\right)+\varepsilon$ for any $x, x^{\prime} \in X_{0}$.

We call such a set $X_{0}$ a nonexceptional domain of $f$.

Lemma 4.8 ([17, Lemma 5.4]). If a function $f: X \rightarrow \mathbb{R}$ on an mm-space $X$ is 1-Lipschitz up to an additive error $\varepsilon \geq 0$, then there exists a 1-Lipschitz function $\tilde{f}: X \rightarrow \mathbb{R}$ such that

$$
d_{\mathrm{KF}}^{m_{X}}(f, \tilde{f}) \leq \varepsilon .
$$

Lemma 4.9 ([17, Lemma 5.27]). Let $X$ and $Y$ be two mm-spaces and $p: X \rightarrow Y$ a Borel measurable map. For two real numbers $\varepsilon, \delta>0$, we consider the two following conditions.

$\left(\mathrm{A}_{\varepsilon}\right) p^{\star} \operatorname{Lip}_{1}(Y) \subset U_{\varepsilon+\eta}\left(\mathcal{L i p}_{1}(X)\right)$ for any $\eta>0$.

$\left(\mathrm{B}_{\delta}\right)$ p is 1-Lipschitz up to $\delta$.

Then we have the following (1) and (2).

(1) There exists a real number $\delta=\delta(Y, \varepsilon)>0$ for any $\varepsilon>0$ such that $\lim _{\varepsilon \rightarrow 0} \delta(Y, \varepsilon)=0$ and if $\left(\mathrm{A}_{\varepsilon}\right)$ holds and if $d_{\mathrm{P}}\left(p_{*} m_{X}, m_{Y}\right)<\varepsilon$, then we have $\left(\mathrm{B}_{\delta}\right)$.

(2) If $\left(\mathrm{B}_{\delta}\right)$ holds, then we have $\left(\mathrm{A}_{\delta}\right)$.

The following lemma gives a condition equivalent to (2) of Theorem 1.1.

Lemma 4.10. Let $F_{n}:[0,+\infty)^{2} \rightarrow[0,+\infty)$ be a function, $n=1,2, \ldots$. Assume that $F_{n}$ uniformly converges to a continuous function $F$ on compact sets. Then the following (1) and (2) are equivalent to each other.

(1) For any $s, t \in[0,+\infty)$,

$$
\lim _{n \rightarrow \infty}\left(F_{n}(s, t)-\inf _{s \leq s^{\prime} ; t \leq t^{\prime}} F_{n}\left(s^{\prime}, t^{\prime}\right)\right)=0 .
$$

(2) For any $D>0$,

$$
\lim _{n \rightarrow \infty} \sup _{0 \leq s, t \leq D}\left(F_{n}(s, t)-\inf _{s \leq s^{\prime} ; t \leq t^{\prime}} F_{n}\left(s^{\prime}, t^{\prime}\right)\right)=0 .
$$

Proof. It is trivial that (2) implies (1). We prove that (1) implies (2). Suppose that the condition (2) does not hold in order to prove the contraposition. There exists a real number $D>0$ such that

$$
\limsup _{n \rightarrow \infty} \sup _{0 \leq s, t \leq D}\left(F_{n}(s, t)-\inf _{s \leq s^{\prime} ; t \leq t^{\prime}} F_{n}\left(s^{\prime}, t^{\prime}\right)\right)>0 .
$$


Choosing a subsequence of $n$, we can assume that there exist a real number $\eta>0$ and a sequence $\left\{\left(s_{n}, t_{n}\right)\right\}_{n \in \mathbb{N}} \subset[0, D]^{2}$ such that

$$
F_{n}\left(s_{n}, t_{n}\right)-\inf _{s_{n} \leq s^{\prime} ; t_{n} \leq t^{\prime}} F_{n}\left(s^{\prime}, t^{\prime}\right)>\eta
$$

Choosing a subsequence again, we can assume that $s_{n}, t_{n}$ converge to $s_{\infty}, t_{\infty}$, respectively, as $n \rightarrow \infty$. We see that $s_{\infty}, t_{\infty} \leq D$. By the continuity of $F$, there exists a real number $\delta>0$ such that

$$
\left|F(s, t)-F\left(s_{\infty}, t_{\infty}\right)\right|<\frac{\eta}{8}
$$

for any $s, t \in[0,+\infty)$ with $\left|s-s_{\infty}\right|,\left|t-t_{\infty}\right| \leq \delta$. Since $F_{n}$ uniformly converges to $F$ on $[0, D+\delta]^{2}$, for every sufficiently large $n$,

$$
\sup _{0 \leq s, t \leq D+\delta}\left|F_{n}(s, t)-F(s, t)\right|<\frac{\eta}{8}
$$

Let $\Gamma:=\left\{(s, t) \in[0,+\infty)^{2}|| s-s_{\infty}|| t-,t_{\infty} \mid \leq \delta\right\}$. For every sufficiently large $n$ and for every $(s, t),\left(s^{\prime}, t^{\prime}\right) \in \Gamma$, we have

$$
\begin{aligned}
& \left|F_{n}(s, t)-F_{n}\left(s^{\prime}, t^{\prime}\right)\right| \\
\leq & \left|F_{n}(s, t)-F(s, t)\right|+\left|F(s, t)-F\left(s_{\infty}, t_{\infty}\right)\right| \\
& +\left|F\left(s_{\infty}, t_{\infty}\right)-F\left(s^{\prime}, t^{\prime}\right)\right|+\left|F\left(s^{\prime}, t^{\prime}\right)-F_{n}\left(s^{\prime}, t^{\prime}\right)\right| \\
< & \frac{\eta}{2} .
\end{aligned}
$$

Let $s_{\star}:=\max \left\{s_{\infty}-\delta, 0\right\}$ and $t_{\star}:=\max \left\{t_{\infty}-\delta, 0\right\}$. Taking into account that $\left(s_{\star}, t_{\star}\right) \in \Gamma$ and $\left(s_{n}, t_{n}\right) \in \Gamma$ for every sufficiently large $n$, we have

$$
F_{n}\left(s_{\star}, t_{\star}\right)-\inf _{s_{\star} \leq s^{\prime} ; t_{\star} \leq t^{\prime}} F_{n}\left(s^{\prime}, t^{\prime}\right)>F_{n}\left(s_{n}, t_{n}\right)-\frac{\eta}{2}-\inf _{s_{n} \leq s^{\prime} ; t_{n} \leq t^{\prime}} F_{n}\left(s^{\prime}, t^{\prime}\right)>\frac{\eta}{2}
$$

for every sufficiently large $n$. This means that the condition (1) does not hold. The proof is completed.

Lemma 4.11. Let $X_{n}, Y_{n}, X$, and $Y$ be $m m$-spaces and let $F_{n}$ and $F$ be functions in $\mathscr{F}^{2}$, where $n=1,2, \ldots$ Assume that $F_{n}$ converges pointwise to $F$ and satisfies the condition (2) of Theorem 1.1. Let $p_{n}: X_{n} \rightarrow X$ and $q_{n}: Y_{n} \rightarrow Y$ be maps. If $p_{n}, q_{n}$ are 1-Lipschitz up to $\varepsilon_{n}, \delta_{n}$ respectively, and both

$$
d_{\mathrm{P}}\left(p_{n \star} m_{X_{n}}, m_{X}\right) \leq \varepsilon_{n} \quad \text { and } \quad d_{\mathrm{P}}\left(q_{n \star} m_{Y_{n}}, m_{Y}\right) \leq \delta_{n}
$$

hold for some sequences $\varepsilon_{n}, \delta_{n} \rightarrow 0$ as $n \rightarrow \infty$, then the map $p_{n} \times q_{n}: X_{n} \times_{F_{n}} Y_{n} \rightarrow X \times_{F} Y$ is 1-Lipschitz up to $\eta_{n}$ for some sequence $\eta_{n} \rightarrow 0$ as $n \rightarrow \infty$.

Proof. The proof is similar to that of Proposition 4.2.

Take any real number $\varepsilon>0$. It suffices to prove that the map $p_{n} \times q_{n}$ is 1 -Lipschitz up to $F(\varepsilon, \varepsilon)+6 \varepsilon$ for any sufficiently large $n \in \mathbb{N}$. By the inner regularity of $m_{X}$ and $m_{Y}$, there exist compact sets $K \subset X$ and $K^{\prime} \subset Y$ such that

$$
m_{X}(K) \geq 1-\varepsilon \quad \text { and } \quad m_{Y}\left(K^{\prime}\right) \geq 1-\varepsilon .
$$

Let $D_{\varepsilon}:=\max \left\{\operatorname{diam} K, \operatorname{diam} K^{\prime}\right\}+2 \varepsilon$. Then, by the assumptions and Lemma 4.10, for any sufficiently large $n \in \mathbb{N}$,

- $p_{n}, q_{n}$ are both 1-Lipschitz up to $\varepsilon$,

- $d_{\mathrm{P}}\left(p_{n \star} m_{X_{n}}, m_{X}\right) \leq \varepsilon$ and $d_{\mathrm{P}}\left(q_{n \star} m_{Y_{n}}, m_{Y}\right) \leq \varepsilon$ hold,

- $\left|F_{n}(s, t)-F(s, t)\right|<\varepsilon$ holds for all $s, t \in\left[0, D_{\varepsilon}\right]$,

- $F_{n}(s, t) \leq F_{n}\left(s^{\prime}, t^{\prime}\right)+\varepsilon$ holds for any $s, t \in\left[0, D_{\varepsilon}\right]$ and for any $s^{\prime}, t^{\prime} \in[0,+\infty)$ with $s \leq s^{\prime}$ and $t \leq t^{\prime}$.

Let $X_{n}^{\prime}, Y_{n}^{\prime}$ be nonexceptional domains of $p_{n}, q_{n}$ respectively and let

$$
\widetilde{X}_{n}:=X_{n}^{\prime} \cap p_{n}^{-1}\left(U_{\varepsilon}(K)\right) \quad \text { and } \quad \tilde{Y}_{n}:=Y_{n}^{\prime} \cap q_{n}^{-1}\left(U_{\varepsilon}\left(K^{\prime}\right)\right) \text {. }
$$


By the similar proof to that of (4.10), we have

$$
m_{X_{n}} \otimes m_{Y_{n}}\left(\widetilde{X}_{n} \times \widetilde{Y}_{n}\right) \geq 1-6 \varepsilon .
$$

For any $(x, y),\left(x^{\prime}, y^{\prime}\right) \in \widetilde{X}_{n} \times \widetilde{Y}_{n}$, we have

$$
\begin{aligned}
& d_{F}\left(\left(\varphi_{n} \times \psi_{n}\right)(x, y),\left(\varphi_{n} \times \psi_{n}\right)\left(x^{\prime}, y^{\prime}\right)\right) \\
= & F\left(d_{X}\left(\varphi_{n}(x), \varphi_{n}\left(x^{\prime}\right)\right), d_{Y}\left(\psi_{n}(y), \psi_{n}\left(y^{\prime}\right)\right)\right) \\
\leq & F_{n}\left(d_{X}\left(\varphi_{n}(x), \varphi_{n}\left(x^{\prime}\right)\right), d_{Y}\left(\psi_{n}(y), \psi_{n}\left(y^{\prime}\right)\right)\right)+\varepsilon \\
\leq & F_{n}\left(d_{X_{n}}\left(x, x^{\prime}\right)+\varepsilon, d_{Y_{n}}\left(y, y^{\prime}\right)+\varepsilon\right)+2 \varepsilon \\
\leq & F_{n}\left(d_{X_{n}}\left(x, x^{\prime}\right), d_{Y_{n}}\left(y, y^{\prime}\right)\right)+F_{n}(\varepsilon, \varepsilon)+2 \varepsilon \\
\leq & d_{F_{n}}\left((x, y),\left(x^{\prime}, y^{\prime}\right)\right)+F(\varepsilon, \varepsilon)+3 \varepsilon,
\end{aligned}
$$

where the first and second inequalities follow from

$$
d_{X}\left(\varphi_{n}(x), \varphi_{n}\left(x^{\prime}\right)\right) \leq D_{\varepsilon} \quad \text { and } \quad d_{Y}\left(\psi_{n}(y), \psi_{n}\left(y^{\prime}\right)\right) \leq D_{\varepsilon} .
$$

Therefore the map $p_{n} \times q_{n}$ is 1-Lipschitz up to $F(\varepsilon, \varepsilon)+6 \varepsilon$. This completes the proof.

Given two subsets $A$ and $B$ of a metric space $X$, we define

$$
d_{X}(A, B):=\inf _{a \in A, b \in B} d_{X}(a, b) .
$$

Definition 4.12 ( $\kappa$-distance). Let $\kappa>0$ and let $X$ be an mm-space. We define the $\kappa$-distance $d_{+}\left(A_{1}, A_{2} ;+\kappa\right)$ between two Borel subsets $A_{1}$ and $A_{2}$ of $X$ as the supremum of $d_{X}\left(B_{1}, B_{2}\right)$ over all Borel subsets $B_{1} \subset A_{1}$ and $B_{2} \subset A_{2}$ with $m_{X}\left(B_{1}\right) \geq \kappa$ and $m_{X}\left(B_{2}\right) \geq \kappa$. We set $d_{+}\left(A_{1}, A_{2} ;+\kappa\right):=0$ if $\min \left\{m_{X}\left(A_{1}\right), m_{X}\left(A_{2}\right)\right\}<\kappa$.

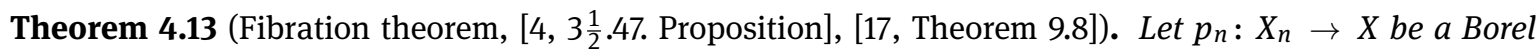
measurable map between mm-spaces $X_{n}$ and $X$, where $n=1,2, \ldots$, such that $d_{\mathrm{P}}\left(p_{n \star} m_{X_{n}}, m_{X}\right)$ tends to 0 as $n \rightarrow \infty$. Then, each $p_{n}$ enforces $\varepsilon_{n}$-concentration of $X_{n}$ to $X$ for some sequence $\varepsilon_{n} \rightarrow 0$ if and only if we have the following (1), (2), and (3).

(1) Each $p_{n}$ is 1-Lipschitz up to some additive error $\varepsilon_{n}^{\prime}$ with $\varepsilon_{n}^{\prime} \rightarrow 0$.

(2) Let $B \subset X$ be an arbitrary Borel subset and let $\mu_{B, n}$ be the probability measure on $X_{n}$ defined by

$$
\mu_{B, n}:=m_{X_{n}}\left(\cdot \cap p_{n}^{-1}(B)\right) / m_{X_{n}}\left(p_{n}^{-1}(B)\right) .
$$

Then, for any $\kappa>0$, we have

$$
\limsup _{n \rightarrow \infty} \operatorname{ObsDiam}\left(\left(p_{n}^{-1}(B), d_{X_{n}}, \mu_{B, n}\right) ;-\kappa\right) \leq \operatorname{diam} B .
$$

(3) For any two Borel subsets $B_{1}, B_{2} \subset X$ and any $\kappa>0$, we have

$$
\limsup _{n \rightarrow \infty} d_{+}\left(p_{n}^{-1}\left(B_{1}\right), p_{n}^{-1}\left(B_{2}\right) ;+\kappa\right) \leq d_{X}\left(B_{1}, B_{2}\right)+\operatorname{diam} B_{1}+\operatorname{diam} B_{2} .
$$

Proposition 4.14. Let $X$ and $Y$ be two metric spaces and let $F$ be a function in $\mathscr{F}^{2}$. If $F$ is an isotone, then

$$
d_{F}\left(A \times B, A^{\prime} \times B^{\prime}\right)=F\left(d_{X}\left(A, A^{\prime}\right), d_{Y}\left(B, B^{\prime}\right)\right)
$$

for any subsets $A, A^{\prime} \subset X$ and $B, B^{\prime} \subset Y$.

Proof. Since $F$ is an isotone, for any $(a, b) \in A \times B$ and $\left(a^{\prime}, b^{\prime}\right) \in A^{\prime} \times B^{\prime}$,

$$
d_{F}\left((a, b),\left(a^{\prime}, b^{\prime}\right)\right)=F\left(d_{X}\left(a, a^{\prime}\right), d_{Y}\left(b, b^{\prime}\right)\right) \geq F\left(d_{X}\left(A, A^{\prime}\right), d_{Y}\left(B, B^{\prime}\right)\right),
$$


which implies $d_{F}\left(A \times B, A^{\prime} \times B^{\prime}\right) \geq F\left(d_{X}\left(A, A^{\prime}\right), d_{Y}\left(B, B^{\prime}\right)\right)$. Let us prove the opposite inequality. We take any two real numbers $\varepsilon, \delta$ such that $d_{X}\left(A, A^{\prime}\right)<\varepsilon$ and $d_{Y}\left(B, B^{\prime}\right)<\delta$. There exist $a \in A, a^{\prime} \in A^{\prime}, b \in B$, and $b^{\prime} \in B^{\prime}$ such that $d_{X}\left(a, a^{\prime}\right)<\varepsilon$ and $d_{Y}\left(b, b^{\prime}\right)<\delta$. Then

$$
d_{F}\left((a, b),\left(a^{\prime}, b^{\prime}\right)\right)=F\left(d_{X}\left(a, a^{\prime}\right), d_{Y}\left(b, b^{\prime}\right)\right) \leq F(\varepsilon, \delta),
$$

which implies $d_{F}\left(A \times B, A^{\prime} \times B^{\prime}\right) \leq F(\varepsilon, \delta)$. By the continuity of $F$, we have $d_{F}\left(A \times B, A^{\prime} \times B^{\prime}\right) \leq$ $F\left(d_{X}\left(A, A^{\prime}\right), d_{Y}\left(B, B^{\prime}\right)\right)$. This completes the proof.

Proof of "(2) $\Rightarrow(1)$ " of Theorem 1.1. The idea of the proof is based on the same of the proof of the fibration theorem.

Assume that the functions $F_{n}$ satisfy the condition (2) and two sequences $\left\{X_{n}\right\}_{n \in \mathbb{N}}$ and $\left\{Y_{n}\right\}_{n \in \mathbb{N}}$ concentrate to $X$ and $Y$ respectively. Note that the function $F$ is an isotone. By Theorem 2.19, there exist Borel measurable maps $p_{n}: X_{n} \rightarrow X$, where $n=1,2, \ldots$, that enforce $\varepsilon_{n}$-concentration of $X_{n}$ to $X$ and $d_{\mathrm{P}}\left(p_{n \star} m_{X_{n}}, m_{X}\right) \leq \varepsilon_{n}$ for some sequence $\varepsilon_{n} \rightarrow 0$. Similarly, there exist Borel measurable maps $q_{n}: Y_{n} \rightarrow Y$, where $n=1,2, \ldots$, that enforce $\varepsilon_{n}$-concentration of $Y_{n}$ to $Y$ and $d_{\mathrm{P}}\left(q_{n \star} m_{Y_{n}}, m_{Y}\right) \leq \varepsilon_{n}$. Since

$$
d_{\mathrm{P}}\left(\left(p_{n} \times q_{n}\right) \star\left(m_{X_{n}} \otimes m_{Y_{n}}\right), m_{X} \otimes m_{Y}\right) \leq \max \left\{2 \varepsilon_{n}, 4 F\left(\varepsilon_{n}, \varepsilon_{n}\right)\right\}
$$

follows from Lemma 4.5, it suffices to prove that the map $p_{n} \times q_{n}$ enforces $\varepsilon_{n}^{\prime}$-concentration of $X_{n} \times F_{n} Y_{n}$ to $X \times_{F} Y$ for some sequence $\varepsilon_{n}^{\prime} \rightarrow 0$. By Lemma 4.9 (1) and Lemma 4.11, the map $p_{n} \times q_{n}$ is 1-Lipschitz up to $\varepsilon_{n}^{\prime}$ for some $\varepsilon_{n}^{\prime} \rightarrow 0$. By Lemma 4.9 (2), we have

$$
\left(p_{n} \times q_{n}\right)^{\star} \mathcal{L i p}_{1}\left(X \times_{F} Y\right) \subset U_{2 \varepsilon_{n}^{\prime}}\left(\mathcal{L i p}_{1}\left(X_{n} \times_{F_{n}} Y_{n}\right)\right) .
$$

Therefore, for any real number $\varepsilon>0$, it suffices to prove that

$$
\operatorname{Lip}_{1}\left(X_{n} \times_{F_{n}} Y_{n}\right) \subset U_{42 F(\varepsilon, \varepsilon)+5 \varepsilon}\left(\left(p_{n} \times q_{n}\right)^{\star} \mathcal{L} \operatorname{Lip}_{1}\left(X \times_{F} Y\right)\right)
$$

holds for every sufficiently large $n$.

We take any $\varepsilon>0$ and any $f_{n} \in \mathcal{L} \operatorname{Lip}_{1}\left(X_{n} \times_{F_{n}} Y_{n}\right)$. There are finitely many mutually disjoint nonempty open subsets $B_{1}^{X}, B_{2}^{X}, \ldots, B_{N}^{X} \subset X$ such that $m_{X}\left(\partial B_{i}^{X}\right)=0$, $\operatorname{diam} B_{i}^{X}<\varepsilon$, and

$$
m_{X}\left(X \backslash \bigcup_{i=1}^{N} B_{i}^{X}\right)<\varepsilon
$$

Similarly, there exist mutually disjoint nonempty open subsets $B_{1}^{Y}, B_{2}^{Y}, \ldots, B_{M}^{Y} \subset Y$ such that $m_{Y}\left(\partial B_{k}^{Y}\right)=0$, $\operatorname{diam} B_{k}^{Y}<\varepsilon$, and

$$
m_{Y}\left(Y \backslash \bigcup_{k=1}^{M} B_{k}^{Y}\right)<\varepsilon .
$$

For each $i=1,2, \ldots, N$ and $k=1,2, \ldots, M$, we take points $x_{i} \in B_{i}^{X}$ and $y_{k} \in B_{k}^{Y}$ and fix them. We put

$$
\begin{array}{ll}
A_{i n}^{X}:=p_{n}^{-1}\left(B_{i}^{X}\right), & \mu_{i n}:=\left.m_{X_{n}}\left(A_{i n}^{X}\right)^{-1} m_{X_{n}}\right|_{A_{i n}^{X}}, \\
A_{k n}^{Y}:=q_{n}^{-1}\left(B_{k}^{Y}\right), & v_{k n}:=\left.m_{Y_{n}}\left(A_{k n}^{Y}\right)^{-1} m_{Y_{n}}\right|_{A_{k n}^{Y}}
\end{array}
$$

for every $i$ and $k$. Note that $m_{X_{n}}\left(A_{i n}^{X}\right)$ converges to $m_{X}\left(B_{i}^{X}\right)$ and $m_{Y_{n}}\left(A_{k n}^{Y}\right)$ converges to $m_{Y}\left(B_{k}^{Y}\right)$ as $n \rightarrow \infty$. We define a Borel measurable map $g_{n}: X \times Y \rightarrow \mathbb{R}$ by

$$
g_{n}(x, y):= \begin{cases}\operatorname{lm}\left(\left.f_{n}\right|_{A_{i n}^{X} \times A_{k n}^{Y}} ; \mu_{i n} \otimes v_{k n}\right) & \text { if }(x, y) \in B_{i}^{X} \times B_{k}^{Y}, \\ 0 & \text { otherwise. }\end{cases}
$$

Our immediate goal is to prove that $g_{n}$ is 1-Lipschitz up to $30 F(\varepsilon, \varepsilon)+2 \varepsilon$ with respect to the metric $d_{F}$ and the measure $\left(p_{n} \times q_{n}\right)_{\star}\left(m_{X_{n}} \otimes m_{Y_{n}}\right)$ for every sufficiently large $n$.

Setting

$$
\rho_{i j k l}:=F\left(d_{X}\left(x_{i}, x_{j}\right), d_{Y}\left(y_{k}, y_{l}\right)\right)+4 F(\varepsilon, \varepsilon)+\varepsilon
$$

for $i, j=1,2, \ldots, N$ and $k, l=1,2, \ldots, M$, we find $\lambda>0$ such that $0<\lambda \rho_{i j k l}<1 / 4$. 
Claim 4.15. For each $i, j, k$, $l$ and every sufficiently large $n$, we have

$$
d_{\mathrm{P}}^{(\lambda)}\left(\mu_{i n} \otimes v_{k n}, \mu_{j n} \otimes v_{l n}\right) \leq \rho_{i j k l},
$$

where $d_{\mathrm{P}}^{(\lambda)}$ in the left-hand side is with respect to $d_{F_{n}}$.

Proof. The proof of the claim is similar to that of [17, Claim 9.9].

We fix $i, j, k$, and $l$. By the $\pi$ - $\lambda$ theorem, it is sufficient to prove that

$$
\mu_{j n} \otimes v_{l n}\left(U_{\rho_{i j k l}}\left(C_{n} \times D_{n}\right)\right) \geq \mu_{i n} \otimes v_{k n}\left(C_{n} \times D_{n}\right)-\lambda \rho_{i j k l}
$$

for any Borel subset $C_{n} \subset X_{n}$ and $D_{n} \subset Y_{n}$. Take any Borel subsets $C_{n} \subset X_{n}$ and $D_{n} \subset Y_{n}$. We can assume that $C_{n} \subset A_{i n}^{X}$ and $D_{n} \subset A_{k n}^{Y}$ since $\mu_{i n}\left(C_{n}\right)=\mu_{i n}\left(C_{n} \cap A_{i n}^{X}\right)$ and $v_{k n}\left(D_{n}\right)=v_{k n}\left(D_{n} \cap A_{k n}^{Y}\right)$. Let $\kappa$ be a real number such that

$$
0<\kappa \leq \lambda \rho_{i j k l} \inf _{n \in \mathbb{N}} \min \left\{m_{X_{n}}\left(A_{i n}^{X}\right), m_{X_{n}}\left(A_{j n}^{X}\right), m_{Y_{n}}\left(A_{k n}^{Y}\right), m_{Y_{n}}\left(A_{l n}^{Y}\right)\right\}
$$

If $m_{X_{n}}\left(C_{n}\right)<\kappa$ or $m_{Y_{n}}\left(D_{n}\right)<\kappa$, then we have

$$
\mu_{i n} \otimes v_{k n}\left(C_{n} \times D_{n}\right)=\frac{m_{X_{n}}\left(C_{n}\right) m_{Y_{n}}\left(D_{n}\right)}{m_{X_{n}}\left(A_{i n}^{X}\right) m_{Y_{n}}\left(A_{k n}^{Y}\right)} \leq \lambda \rho_{i j k l},
$$

so that we obtain (4.17). Assume that $m_{X_{n}}\left(C_{n}\right) \geq \kappa$ and $m_{Y_{n}}\left(D_{n}\right) \geq \kappa$. We define two functions $\varphi_{n}: A_{j n}^{X} \rightarrow \mathbb{R}$ and $\psi_{n}: A_{l n}^{Y} \rightarrow \mathbb{R}$ by

$$
\varphi_{n}(x):=d_{X_{n}}\left(x, C_{n}\right) \text { and } \psi_{n}(y):=d_{Y_{n}}\left(y, D_{n}\right)
$$

for $x \in A_{j n}^{X}$ and $y \in A_{l n}^{Y}$, and let

$$
\begin{aligned}
& E_{n}^{X}:=\left\{x \in A_{j n}^{X}|| \varphi_{n}(x)-\operatorname{lm}\left(\varphi_{n} ; \mu_{j n}\right) \mid \leq \varepsilon\right\}, \\
& E_{n}^{Y}:=\left\{y \in A_{l n}^{Y}|| \psi_{n}(x)-\operatorname{lm}\left(\psi_{n} ; v_{l n}\right) \mid \leq \varepsilon\right\} .
\end{aligned}
$$

For any $\kappa^{\prime} \in(0,1 / 2)$ and every sufficiently large $n$, by Theorem 4.13, we have

$$
\begin{aligned}
& \operatorname{ObsDiam}\left(\mu_{j n} ;-\kappa^{\prime}\right)\left(:=\operatorname{ObsDiam}\left(\left(A_{j n}^{X}, \mu_{j n}\right) ;-\kappa^{\prime}\right)\right)<\varepsilon, \\
& \operatorname{ObsDiam}\left(\nu_{l n} ;-\kappa^{\prime}\right)\left(:=\operatorname{ObsDiam}\left(\left(A_{l n}^{Y}, \mu_{l n}\right) ;-\kappa^{\prime}\right)\right)<\varepsilon,
\end{aligned}
$$

and then, by Lemma 2.26, we have

$$
\operatorname{LeRad}\left(\mu_{j n} ;-\kappa^{\prime}\right)<\varepsilon \text { and } \operatorname{LeRad}\left(\nu_{l n} ;-\kappa^{\prime}\right)<\varepsilon .
$$

Thus we have $\mu_{j n}\left(E_{n}^{X}\right), v_{l n}\left(E_{n}^{Y}\right) \rightarrow 1$ as $n \rightarrow \infty$, which imply

$$
m_{X_{n}}\left(E_{n}^{X}\right) \geq \kappa, m_{Y_{n}}\left(E_{n}^{Y}\right) \geq \kappa \text {, and } \mu_{j n} \otimes v_{l n}\left(E_{n}^{X} \times E_{n}^{Y}\right) \geq 1-\lambda \rho_{i j k l}
$$

for every sufficiently large $n$. By Theorem 4.13, it holds that

$$
d_{X_{n}}\left(C_{n}, E_{n}^{X}\right) \leq d_{+}\left(A_{i n}^{X}, A_{j n}^{X} ;+\kappa\right)<d_{X}\left(B_{i}, B_{j}\right)+2 \varepsilon \leq d_{X}\left(x_{i}, x_{j}\right)+2 \varepsilon
$$

for every sufficiently large $n$. For any two points $x, x^{\prime} \in E_{n}^{X}$, we have

$$
d_{X_{n}}\left(x, C_{n}\right) \leq \operatorname{lm}\left(\varphi_{n} ; \mu_{j n}\right)+\varepsilon \leq d_{X_{n}}\left(x^{\prime}, C_{n}\right)+2 \varepsilon,
$$

which implies $d_{X_{n}}\left(x, C_{n}\right) \leq d_{X_{n}}\left(E_{n}^{X}, C_{n}\right)+2 \varepsilon<d_{X}\left(x_{i}, x_{j}\right)+4 \varepsilon$ for every $x \in E_{n}^{X}$. Similarly, $d_{Y_{n}}\left(y, D_{n}\right)<$ $d_{Y}\left(y_{k}, y_{l}\right)+4 \varepsilon$ also holds for every $y \in E_{n}^{Y}$. By Proposition 4.14, we have

$$
\begin{aligned}
& d_{F_{n}}\left((x, y), C_{n} \times D_{n}\right) \\
& =F_{n}\left(d_{X_{n}}\left(x, C_{n}\right), d_{Y_{n}}\left(y, D_{n}\right)\right) \\
& <F\left(d_{X_{n}}\left(x, C_{n}\right), d_{Y_{n}}\left(y, D_{n}\right)\right)+\varepsilon \\
& \leq F\left(d_{X}\left(x_{i}, x_{j}\right)+4 \varepsilon, d_{Y}\left(y_{k}, y_{l}\right)+4 \varepsilon\right)+\varepsilon \\
& \leq F\left(d_{X}\left(x_{i}, x_{j}\right), d_{Y}\left(y_{k}, y_{l}\right)\right)+4 F(\varepsilon, \varepsilon)+\varepsilon=\rho_{i j k l}
\end{aligned}
$$


for any $(x, y) \in E_{n}^{X} \times E_{n}^{Y}$ and every sufficiently large $n$, where the first inequality follows from the fact that $F_{n}$ uniformly converges to $F$ on any compact sets, and the second follows from the fact that $F$ is an isotone. This means $E_{n}^{X} \times E_{n}^{Y} \subset U_{\rho_{i j k l}}\left(C_{n} \times D_{n}\right)$. Therefore we have

$$
\begin{aligned}
& \mu_{j n} \otimes v_{l n}\left(U_{\rho_{i j k l}}\left(C_{n} \times D_{n}\right)\right) \geq \mu_{j n} \otimes v_{l n}\left(E_{n}^{X} \times E_{n}^{Y}\right) \\
& \geq 1-\lambda \rho_{i j k l} \geq \mu_{i n} \otimes v_{k n}\left(C_{n} \times D_{n}\right)-\lambda \rho_{i j k l},
\end{aligned}
$$

so that we obtain (4.17). This completes the proof.

By Claim 4.15 and Strassen's theorem (Theorem 2.22), there exists a $\rho_{i j k l}$-subtransport plan $\pi_{i j k l}^{n}$ between $\mu_{i n} \otimes v_{k n}$ and $\mu_{j n} \otimes v_{l n}$ such that def $\pi_{i j k l}^{n} \leq \lambda \rho_{i j k l}$. Since def $\pi_{i j k l}^{n}<1 / 4$, we have

$$
\begin{aligned}
& \left|g_{n}(x, y)-g_{n}\left(x^{\prime}, y^{\prime}\right)\right| \\
& =\left|\operatorname{lm}\left(\left.f_{n}\right|_{A_{i n}^{X} \times A_{k n}^{Y}} ; \mu_{i n} \otimes v_{k n}\right)-\operatorname{lm}\left(\left.f_{n}\right|_{A_{j n}^{X} \times A_{l n}^{Y}} ; \mu_{j n} \otimes v_{l n}\right)\right| \\
& \leq \rho_{i j k l}+\operatorname{ObsDiam}\left(\mu_{i n} \otimes v_{k n} ;-\frac{1}{4}\right)+\operatorname{ObsDiam}\left(\mu_{j n} \otimes v_{l n} ;-\frac{1}{4}\right)
\end{aligned}
$$

for any $(x, y) \in B_{i}^{X} \times B_{k}^{Y}$ and any $\left(x^{\prime}, y^{\prime}\right) \in B_{j}^{X} \times B_{l}^{Y}$, where the last inequality follows from Lemma 2.27. We have

$$
\begin{aligned}
& \rho_{i j k l}=F\left(d_{X}\left(x_{i}, x_{j}\right), d_{Y}\left(y_{k}, y_{l}\right)\right)+4 F(\varepsilon, \varepsilon)+\varepsilon \\
& \leq F\left(d_{X}\left(x, x^{\prime}\right)+2 \varepsilon, d_{Y}\left(y, y^{\prime}\right)+2 \varepsilon\right)+4 F(\varepsilon, \varepsilon)+\varepsilon \\
& \leq d_{F}\left((x, y),\left(x^{\prime}, y^{\prime}\right)\right)+6 F(\varepsilon, \varepsilon)+\varepsilon,
\end{aligned}
$$

where the first inequality follows from the fact that $F$ is an isotone. Moreover, by Lemma 3.27, we have

$$
\begin{aligned}
& \operatorname{ObsDiam}\left(\mu_{i n} \otimes v_{k n} ;-\frac{1}{4}\right) \\
& \leq 4 F\left(\operatorname{ObsDiam}\left(\mu_{i n} ;-\frac{1}{16}\right), 0\right)+8 F\left(0, \operatorname{ObsDiam}\left(v_{k n} ;-\frac{1}{16}\right)\right) \\
& \leq 12 F\left(\operatorname{ObsDiam}\left(\mu_{i n} ;-\frac{1}{16}\right), \operatorname{ObsDiam}\left(v_{k n} ;-\frac{1}{16}\right)\right),
\end{aligned}
$$

and, by Theorem 4.13, we see that

$$
\operatorname{ObsDiam}\left(\mu_{i n} ;-\frac{1}{16}\right)<\varepsilon \quad \text { and } \operatorname{ObsDiam}\left(v_{k n} ;-\frac{1}{16}\right)<\varepsilon
$$

for every sufficiently large $n$. Thus we obtain

$$
\left|g_{n}(x, y)-g_{n}\left(x^{\prime}, y^{\prime}\right)\right| \leq d_{F}\left((x, y),\left(x^{\prime}, y^{\prime}\right)\right)+30 F(\varepsilon, \varepsilon)+\varepsilon
$$

for any $(x, y),\left(x^{\prime}, y^{\prime}\right) \in \bigcup_{i=1}^{N} \bigcup_{k=1}^{M} B_{i}^{X} \times B_{k}^{Y}$ and every sufficiently large $n$. Furthermore it holds that

$$
\begin{aligned}
& \lim _{n \rightarrow \infty}\left(p_{n} \times q_{n}\right)_{\star}\left(m_{X_{n}} \otimes m_{Y_{n}}\right)\left(\bigcup_{i=1}^{N} \bigcup_{k=1}^{M} B_{i}^{X} \times B_{k}^{Y}\right) \\
& =m_{X} \otimes m_{Y}\left(\bigcup_{i=1}^{N} \bigcup_{k=1}^{M} B_{i}^{X} \times B_{k}^{Y}\right)=m_{X}\left(\bigcup_{i=1}^{N} B_{i}^{X}\right) m_{Y}\left(\bigcup_{k=1}^{M} B_{k}^{Y}\right) \\
& \geq(1-\varepsilon)^{2}>1-2 \varepsilon,
\end{aligned}
$$

where the first equality follows from (4.12). Combining this and (4.18) implies that $g_{n}$ is 1-Lipschitz up to $30 F(\varepsilon, \varepsilon)+2 \varepsilon$ with respect to $d_{F}$ and $\left(p_{n} \times q_{n}\right)_{*}\left(m_{X_{n}} \otimes m_{Y_{n}}\right)$ for every sufficiently large $n$. By Lemma 4.8, we see that there exists $\tilde{g}_{n} \in \mathcal{L} i p_{1}\left(X \times_{F} Y\right)$ such that

$$
d_{\mathrm{KF}}^{m_{X_{n}} \otimes m_{Y_{n}}}\left(\left(p_{n} \times q_{n}\right)^{\star} g_{n},\left(p_{n} \times q_{n}\right)^{\star} \tilde{g}_{n}\right) \leq 30 F(\varepsilon, \varepsilon)+2 \varepsilon .
$$


Let $\kappa:=\min \{\varepsilon / N M, 1 / 4\}$. For every sufficiently large $n$, we have

$$
\operatorname{LeRad}\left(\mu_{i n} \otimes v_{k n} ;-\kappa\right) \leq \operatorname{ObsDiam}\left(\mu_{i n} \otimes v_{k n} ;-\kappa\right) \leq 12 F(\varepsilon, \varepsilon)
$$

for each $i=1,2, \ldots, N$ and $k=1,2, \ldots, M$. Setting

$$
K_{i k n}:=\left\{(x, y)|| f_{n}(x, y)-\operatorname{lm}\left(\left.f_{n}\right|_{A_{i n}^{X} \times A_{k n}^{Y}} ; \mu_{i n} \otimes v_{k n}\right) \mid>12 F(\varepsilon, \varepsilon)\right\},
$$

we have

$$
\begin{aligned}
& m_{X_{n}} \otimes m_{Y_{n}}\left(\left|f_{n}-\left(p_{n} \times q_{n}\right)^{\star} g_{n}\right|>12 F(\varepsilon, \varepsilon)\right) \\
& \leq \sum_{i=1}^{N} \sum_{k=1}^{M} m_{X_{n}} \otimes m_{Y_{n}}\left(\left(A_{i n}^{X} \times A_{k n}^{Y}\right) \cap K_{i k n}\right)+m_{X_{n}} \otimes m_{Y_{n}}\left(\left(X_{n} \times Y_{n}\right) \backslash \bigcup_{i=1}^{N} \bigcup_{k=1}^{M} A_{i n}^{X} \times A_{k n}^{Y}\right) \\
& \leq N M \kappa+\left(p_{n} \times q_{n}\right)_{\star}\left(m_{X_{n}} \otimes m_{Y_{n}}\right)\left((X \times Y) \backslash \bigcup_{i=1}^{N} \bigcup_{k=1}^{M} B_{i}^{X} \times B_{k}^{Y}\right) \\
& \leq \varepsilon+2 \varepsilon=3 \varepsilon .
\end{aligned}
$$

Thus we obtain

$$
d_{\mathrm{KF}}^{m_{X_{n}} \otimes m_{Y_{n}}}\left(f_{n},\left(p_{n} \times q_{n}\right)^{\star} g_{n}\right)<12 F(\varepsilon, \varepsilon)+3 \varepsilon,
$$

which implies

$$
d_{\mathrm{KF}}^{m_{X_{n}} \otimes m_{Y_{n}}}\left(f_{n},\left(p_{n} \times q_{n}\right)^{\star} \tilde{g}_{n}\right)<42 F(\varepsilon, \varepsilon)+5 \varepsilon .
$$

This completes the proof.

Proof of Corollary 1.3. We just apply Theorem 1.1 with $F_{n}=F_{p_{n}}, n=1,2, \ldots$, and $F=F_{p}$, where $F_{p}$ is the function of Example 3.7 (1).

Proof of (2) $\Rightarrow$ (1) of Theorem 1.4. Let $Y_{n}, n=1,2, \ldots$, and $Y$ be one-point mm-spaces and let $G_{n}$ and $G$ be the functions on $[0,+\infty)^{2}$ defined by

$$
G_{n}(s, t):=F_{n}(s)+t \quad \text { and } \quad G(s, t):=F(s)+t
$$

for $s, t \in[0,+\infty)$. We apply “(2) $\Rightarrow(1)$ ” of Theorem 1.1.

\subsection{A new specific example of the concentration}

Example 4.16. We consider the $n$-dimensional unit sphere $S^{n}(1)$ and the interval $[0, \pi]$. These spaces are both equipped with the distance and normalized measure induced by the standard Riemannian metric. We take an arbitrary point $\bar{x} \in S^{n}(1)$ and fix it. We attach the interval $[0, \pi]$ to the sphere $S^{n}(1)$ at their points $\pi \in[0, \pi]$ and $\bar{x} \in S^{n}(1)$, and denote their united space by $X_{n}$. That is, the space $X_{n}$ is defined as the $m m$-space

$$
X_{n}:=[0, \pi] \sqcup S^{n}(1) /_{\pi=\bar{x}},
$$

where the distance $d_{X_{n}}$ is defined by

$$
d_{X_{n}}\left(x, x^{\prime}\right):= \begin{cases}d_{[0, \pi]}\left(x, x^{\prime}\right) & \text { if } x, x^{\prime} \in[0, \pi], \\ d_{S^{n}(1)}\left(x, x^{\prime}\right) & \text { if } x, x^{\prime} \in S^{n}(1), \\ d_{[0, \pi]}(x, \pi)+d_{S^{n}(1)}\left(\bar{x}, x^{\prime}\right) & \text { if } x \in[0, \pi], x^{\prime} \in S^{n}(1), \\ d_{[0, \pi]}\left(x^{\prime}, \pi\right)+d_{S^{n}(1)}(\bar{x}, x) & \text { if } x^{\prime} \in[0, \pi], x \in S^{n}(1)\end{cases}
$$

for $x, x^{\prime} \in X_{n}$, and the measure $m_{X_{n}}$ is defined by

$$
m_{X_{n}}:=\frac{1}{2} m_{[0, \pi]}+\frac{1}{2} m_{S^{n}(1)} .
$$


The sequence $\left\{X_{n}\right\}_{n \in \mathbb{N}}$ concentrates to the following mm-space $X$. The mm-space $X$ is the subset $[0, \pi] \cup$ $\{3 \pi / 2\}$ of the one-dimensional Euclidean space $\mathbb{R}$ with the Euclidean distance and the measure

$$
m_{X}:=\frac{1}{2} m_{[0, \pi]}+\frac{1}{2} \delta_{\frac{3}{2} \pi},
$$

where $\delta_{x}$ is the Dirac measure at a point $x$. This is proved by applying Theorem 2.19 to the maps $p_{n}: X_{n} \rightarrow X$, $n=1,2, \ldots$, defined by

$$
p_{n}(x):= \begin{cases}x & \text { if } x \in[0, \pi] \\ \frac{3}{2} \pi & \text { if } x \in S^{n}(1)\end{cases}
$$

If the reader wishes to prove its details, one reads Section 5. The proof is similar to that of Claim 5.1 and 5.3 (and is easier than them).

Applying the implication "(2) $\Rightarrow$ (1)" of Theorem 1.1, we understand the concentration of product spaces of two copies of $X_{n}$ in Example 4.16.

Example 4.17. Let $X_{n}, n=1,2, \ldots$, and $X$ be mm-spaces of Example 4.16. Corollary 1.3 implies that the sequence of the $l_{p}$-product spaces $\left\{X_{n} \times_{p} X_{n}\right\}_{n \in \mathbb{N}}$ concentrates to the $l_{p}$-product space $X \times_{p} X$ for any $p \in$ $[1,+\infty]$. The limit space $X \times_{p} X$ is $\mathrm{mm}$-isomorphic to the subset

$$
\left\{(x, y) \in \mathbb{R}^{2} \mid x, y \in[0, \pi] \cup\{3 \pi / 2\}\right\}
$$

of the $l_{p}$-normed space $\left(\mathbb{R}^{2},\|\cdot\|_{p}\right)$.

\section{The necessity of the isotonicity}

In this section, we prove "(1) $\Rightarrow$ (2)" of Theorem 1.1 and Theorem 1.4. In order to prove them, we construct some counterexample of the condition (1) if the condition (2) does not hold.

We first prove Theorem 1.4.

Proof of "(1) $\Rightarrow$ (2)" of Theorem 1.4. Assume that the condition (2) does not hold. That is, up to choosing a subsequence of $n$, we can assume that there exist two real numbers $s, \eta>0$ and a sequence $\left\{s_{n}\right\}_{n \in \mathbb{N}} \subset$ $(0,+\infty)$ such that

$$
s<s_{n} \quad \text { and } \quad F_{n}(s)>F_{n}\left(s_{n}\right)+\eta
$$

for any $n \in \mathbb{N}$. Moreover, we can assume that

$$
F_{n}\left(s_{n}\right)=\min _{s \leq t \leq s_{n}} F_{n}(t) .
$$

Choosing a subsequence of $n$, we can assume that there exists a limit of $\left\{F_{n}\left(s_{n}\right)\right\}_{n \in \mathbb{N}}$ as $n \rightarrow \infty$. we see that

$$
F(s) \geq \lim _{n \rightarrow \infty} F_{n}\left(s_{n}\right)+\eta .
$$

We define an mm-space $X$ as

$$
X:=\left(\left\{x_{0}, x_{1}\right\}, d_{X}, \frac{1}{2} \delta_{x_{0}}+\frac{1}{2} \delta_{x_{1}}\right), \quad d_{X}\left(x_{0}, x_{1}\right):=s .
$$

We set

$$
r_{n}:=\frac{\sqrt{s_{n}^{2}-s^{2}}}{2}>0 \quad \text { and } \quad k_{n}:=\max \left\{n,\left\lceil r_{n}^{4}\right\rceil\right\}
$$

for each $n$, where $\lceil\cdot\rceil$ is the ceiling function. Let $S^{k_{n}}\left(r_{n}\right)$ be the $k_{n}$-dimensional sphere of radius $r_{n}$ in $\mathbb{R}^{k_{n}+1}$ centered at the origin. The sphere $S^{k_{n}}\left(r_{n}\right)$ is equipped with the Euclidean distance $\|\cdot\|$ and the normalized probability volume measure $\sigma^{k_{n}}$. Define an mm-space $X_{n}$ for each $n$ as

$$
X_{n}:=X \times_{2}\left(S^{k_{n}}\left(r_{n}\right),\|\cdot\|, \sigma^{k_{n}}\right) .
$$


Note that embedding $X$ into the 1-dimensional Euclidean space, $X_{n}$ is regarded as a subset of the $\left(k_{n}+2\right)$ dimensional Euclidean space $\left(\mathbb{R}^{k_{n}+2},\|\cdot\|\right)$ naturally. By Corollary 1.3 and Example 3.28, the sequence $\left\{X_{n}\right\}_{n \in \mathbb{N}}$ concentrates to $X$. Let us prove the following claim.

Claim 5.1. The sequence $\left\{\left(X_{n}, F_{n} \circ d_{X_{n}}, m_{X_{n}}\right)\right\}_{n \in \mathbb{N}}$ concentrates to the mm-space $Y$ defined by

$$
Y:=\left(\left\{y_{0}, y_{1}\right\}, d_{Y}, \frac{1}{2} \delta_{y_{0}}+\frac{1}{2} \delta_{y_{1}}\right), \quad d_{Y}\left(y_{0}, y_{1}\right):=\lim _{n \rightarrow \infty} F_{n}\left(s_{n}\right)
$$

as $n \rightarrow \infty$.

Proof. For $i=0,1$, we set a subset $S_{i}^{n}$ of $X_{n}$ and a measure $\sigma_{i}^{n}$ on $S_{i}^{n}$ by

$$
S_{i}^{n}:=\left\{x_{i}\right\} \times S^{k_{n}}\left(r_{n}\right) \subset X_{n} \subset \mathbb{R}^{k_{n}+2}, \quad \sigma_{i}^{n}:=\delta_{x_{i}} \otimes \sigma^{k_{n}} .
$$

Note that $\left(S_{i}^{n},\|\cdot\|, \sigma_{i}^{n}\right)$ is mm-isomorphic to $\left(S^{k_{n}}\left(r_{n}\right),\|\cdot\|, \sigma^{k_{n}}\right)$ for both $i=0$, 1. Let $p_{n}: X_{n} \rightarrow Y$ be the map defined by

$$
p_{n}(x):= \begin{cases}y_{0} & \text { if } x \in S_{0}^{n}, \\ y_{1} & \text { if } x \in S_{1}^{n}\end{cases}
$$

Note that $p_{n \star} m_{X_{n}}=m_{Y}$. Let $\varepsilon>0$ be a sufficiently small arbitrary real number. We find a number $N \in \mathbb{N}$ such that

$$
\begin{aligned}
& \left|F_{n}(\varepsilon)-F(\varepsilon)\right|<\varepsilon, \quad\left|F_{n}\left(s_{n}\right)-d_{Y}\left(y_{0}, y_{1}\right)\right|<\varepsilon, \\
& \text { and } \operatorname{ObsDiam}\left(\left(S_{i}^{n},\|\cdot\|, \sigma_{i}^{n}\right) ;-\varepsilon\right)<\varepsilon
\end{aligned}
$$

hold for any $n \geq N$ and $i=0,1$. Let us prove that $p_{n}$ enforces $(24 F(\varepsilon)+27 \varepsilon)$-concentration of $\left(X_{n}, F_{n} \circ d_{X_{n}}, m_{X_{n}}\right)$ to $Y$ for any $n \geq N$. We fix $n$ with $n \geq N$. For any $x \in S_{0}^{n}$ and $x^{\prime} \in S_{1}^{n}$, it holds that

$$
s \leq\left\|x-x^{\prime}\right\| \leq s_{n},
$$

so that

$$
F_{n}\left(\left\|x-x^{\prime}\right\|\right) \geq F_{n}\left(s_{n}\right)>d_{Y}\left(y_{0}, y_{1}\right)-\varepsilon,
$$

where the first inequality follows from (5.1). Thus, the map $p_{n}$ is 1-Lipschitz up to $\varepsilon$ with respect to $F_{n} \circ d_{X_{n}}$. By Lemma 4.9, we have

$$
p_{n}{ }^{*} \mathcal{L} i p_{1}(Y) \subset U_{2 \varepsilon}\left(\mathcal{L} i p_{1}\left(X_{n}\right)\right) .
$$

We prove the other side inclusion. We take any function $f_{n} \in \mathcal{L} i p_{1}\left(X_{n}, F_{n} \circ d_{X_{n}}\right)$ and define a function $g_{n}$ : $Y \rightarrow \mathbb{R}$ by

$$
g_{n}\left(y_{0}\right):=\operatorname{lm}\left(f_{n} ; \sigma_{0}^{n}\right), \quad g_{n}\left(y_{1}\right):=\operatorname{lm}\left(f_{n} ; \sigma_{1}^{n}\right) .
$$

By Lemma 2.26 and Lemma 3.22,

$$
\begin{aligned}
& \operatorname{LeRad}\left(\left(S_{i}^{n}, F_{n} \circ\|\cdot\|, \sigma_{i}^{n}\right) ;-2 \varepsilon\right) \\
& \leq \operatorname{ObsDiam}\left(\left(S_{i}^{n}, F_{n} \circ\|\cdot\|, \sigma_{i}^{n}\right) ;-2 \varepsilon\right) \\
& \leq 4 F_{n}\left(\operatorname{ObsDiam}\left(\left(S_{i}^{n},\|\cdot\|, \sigma_{i}^{n}\right) ;-\varepsilon\right)\right) \leq 8 F_{n}(\varepsilon) .
\end{aligned}
$$

Thus we have

$$
\begin{aligned}
& m_{X_{n}}\left\{x \in X_{n}|| f_{n}(x)-p_{n}{ }^{\star} g_{n}(x) \mid>8 F_{n}(\varepsilon)\right\} \\
& =\frac{1}{2} \sum_{i=0}^{1} \sigma_{i}^{n}\left\{x \in S_{i}^{n}|| f_{n}(x)-\operatorname{lm}\left(f_{n} ; \sigma_{i}^{n}\right) \mid>8 F_{n}(\varepsilon)\right\} \leq 2 \varepsilon,
\end{aligned}
$$

which implies $d_{\mathrm{KF}}^{m_{X_{n}}}\left(f_{n}, p_{n}{ }^{\star} g_{n}\right)<8 F_{n}(\varepsilon)+2 \varepsilon<8 F(\varepsilon)+10 \varepsilon$. Let $T_{n}: S_{0}^{n} \rightarrow S_{1}^{n}$ be the map defined by

$$
T_{n}\left(x_{0}, a\right):=\left(x_{1},-a\right)
$$


for $a \in S^{k_{n}}\left(r_{n}\right)$. Note that $T_{n \star} \sigma_{0}^{n}=\sigma_{1}^{n}$. For any $x \in S_{0}^{n}$, we have

$$
\left\|x-T_{n}(x)\right\|^{2}=s^{2}+\left(2 r_{n}\right)^{2}=s_{n}^{2},
$$

so that

$$
F_{n}\left(\left\|x-T_{n}(x)\right\|\right)=F_{n}\left(s_{n}\right) .
$$

Thus, the measure (id, $\left.T_{n}\right)_{\star} \sigma_{0}^{n}$ is an $F_{n}\left(s_{n}\right)$-(sub)transport plan between $\sigma_{0}^{n}$ and $\sigma_{1}^{n}$ (with $\operatorname{def}\left(\left(\mathrm{id}, T_{n}\right)_{\star} \sigma_{0}^{n}\right)=0$ ). By Lemma 2.27 and Lemma 3.22, we have

$$
\begin{aligned}
& \left|g_{n}\left(y_{0}\right)-g_{n}\left(y_{1}\right)\right|=\left|\operatorname{lm}\left(f_{n} ; \sigma_{0}^{n}\right)-\operatorname{lm}\left(f_{n} ; \sigma_{1}^{n}\right)\right| \\
& \leq F_{n}\left(s_{n}\right)+\sum_{i=0}^{1} \operatorname{ObsDiam}\left(\left(S_{i}^{n}, F_{n} \circ\|\cdot\|, \sigma_{i}^{n}\right) ;-2 \varepsilon\right) \\
& \leq F_{n}\left(s_{n}\right)+\sum_{i=0}^{1} 4 F_{n}\left(\operatorname{ObsDiam}\left(\left(S_{i}^{n},\|\cdot\|, \sigma_{i}^{n}\right) ;-\varepsilon\right)\right) \\
& \leq F_{n}\left(s_{n}\right)+16 F_{n}(\varepsilon)<d_{Y}\left(y_{0}, y_{1}\right)+16 F(\varepsilon)+17 \varepsilon .
\end{aligned}
$$

Lemma 4.8 implies $d_{\mathrm{KF}}^{m_{X}}\left(g_{n}\right.$, Lip $\left._{1}(Y)\right)<16 F(\varepsilon)+17 \varepsilon$. Taking

$$
d_{\mathrm{KF}}^{m_{Y}}\left(g_{n}, \mathcal{L i} p_{1}(Y)\right)=d_{\mathrm{KF}}^{m_{X_{n}}}\left(p_{n}{ }^{*} g_{n}, p_{n}{ }^{*} \mathcal{L} i p_{1}(Y)\right)
$$

into account, we have

$$
d_{\mathrm{KF}}^{m_{X_{n}}}\left(f_{n}, p_{n}{ }^{\star} \mathcal{L i p}_{1}(Y)\right) \leq d_{\mathrm{KF}}^{m_{X_{n}}}\left(f_{n}, p_{n}{ }^{*} g_{n}\right)+d_{\mathrm{KF}}^{m_{Y}}\left(g_{n}, \mathcal{L} i p_{1}(Y)\right)<24 F(\varepsilon)+27 \varepsilon .
$$

Thus we obtain $\operatorname{Lip}_{1}\left(X_{n}\right) \subset U_{24 F(\varepsilon)+27 \varepsilon}\left(p_{n}{ }^{*} \mathcal{L i p}_{1}(Y)\right)$ and then the map $p_{n}$ enforces $(24 F(\varepsilon)+27 \varepsilon)$ concentration of $\left(X_{n}, F_{n} \circ d_{X_{n}}, m_{X_{n}}\right)$ to $Y$ for every $n \geq N$. By Theorem 2.19, the sequence $\left\{\left(X_{n}, F_{n} \circ\right.\right.$ $\left.\left.d_{X_{n}}, m_{X_{n}}\right)\right\}_{n \in \mathbb{N}}$ concentrates to $Y$ as $n \rightarrow \infty$. The proof of the claim is now completed.

Since

$$
d_{X}\left(x_{0}, x_{1}\right)=F(s) \geq \lim _{n \rightarrow \infty} F_{n}\left(s_{n}\right)+\eta=d_{Y}\left(y_{0}, y_{1}\right)+\eta,
$$

the mm-space $\left(X, F \circ d_{X}, m_{X}\right)$ is not mm-isomorphic to $Y$. Thus Claim 5.1 means that the condition (1) of Theorem 1.4 does not hold. Therefore we obtain“(1) $\Rightarrow$ (2)" of Theorem 1.4.

We next prove Theorem 1.1. The idea of the proof is same as that of the above proof but the following proof is more complicated.

Proof of "(1) $\Rightarrow$ (2)" of Theorem 1.1. Assume that the condition (2) does not hold. Up to choosing a subsequence of $n$, we can assume that there exist a real number $\eta>0$, a pair $(s, t) \in[0,+\infty)^{2}$, and a sequence $\left\{\left(s_{n}, t_{n}\right)\right\}_{n \in \mathbb{N}} \subset[0,+\infty)^{2}$ such that

$$
s<s_{n}, \quad t<t_{n}, \quad \text { and } \quad F_{n}(s, t)>F_{n}\left(s_{n}, t_{n}\right)+\eta
$$

for any $n \in \mathbb{N}$. We define two mm-spaces $X$ and $Y$ as

$$
\begin{array}{ll}
X:=\left(\left\{\bar{x}_{0}, \bar{x}_{1}\right\}, d_{X}, \frac{1}{2} \delta_{\bar{x}_{0}}+\frac{1}{2} \delta_{\bar{x}_{1}}\right), & d_{X}\left(\bar{x}_{0}, \bar{x}_{1}\right):=s, \\
Y:=\left(\left\{\bar{y}_{0}, \bar{y}_{1}\right\}, d_{Y}, \frac{1}{2} \delta_{\bar{y}_{0}}+\frac{1}{2} \delta_{\bar{y}_{1}}\right), & d_{Y}\left(\bar{y}_{0}, \bar{y}_{1}\right):=t .
\end{array}
$$

For each $n$, let

$$
\begin{aligned}
r_{n}:=\frac{\sqrt{s_{n}^{2}-s^{2}}}{2}>0, & \rho_{n}:=\frac{\sqrt{t_{n}^{2}-t^{2}}}{2}>0, \\
k_{n}:=2 \max \left\{n,\left\lceil r_{n}^{4}\right\rceil\right\}+1, & l_{n}:=2 \max \left\{n,\left\lceil\rho_{n}^{4}\right\rceil\right\}+1 .
\end{aligned}
$$


Define two mm-spaces $X_{n}$ and $Y_{n}$ for each $n$ as

$$
X_{n}:=X \times_{2}\left(S^{k_{n}}\left(r_{n}\right),\|\cdot\|, \sigma^{k_{n}}\right) \quad \text { and } \quad Y_{n}:=Y \times_{2}\left(S^{l_{n}}\left(\rho_{n}\right),\|\cdot\|, \sigma^{l_{n}}\right) .
$$

Note that we regard $X_{n}$ and $Y_{n}$ as subsets of the Euclidean spaces with dimensions $k_{n}+2$ and $l_{n}+2$ respectively, and that the two sequences $\left\{X_{n}\right\}_{n \in \mathbb{N}}$ and $\left\{Y_{n}\right\}_{n \in \mathbb{N}}$ concentrate to $X$ and $Y$ respectively.

In addition, we define three numbers $\alpha_{n}, \beta_{n}, \gamma_{n}$ for each $n$ by

$$
\alpha_{n}:=\min _{\substack{s \leq u_{1} \leq s_{n}, 0 \leq v_{1} \leq 2 \rho_{n}}} F_{n}\left(u_{1}, v_{1}\right), \quad \beta_{n}:=\min _{\substack{0 \leq u_{2} \leq 2 r_{n} \\ t \leq v_{2} \leq t_{n}}} F_{n}\left(u_{2}, v_{2}\right), \quad \gamma_{n}:=\min _{\substack{s \leq u_{3} \leq s_{n} \\ t \leq v_{3} \leq t_{n}}} F_{n}\left(u_{3}, v_{3}\right) \text {. }
$$

Claim 5.2. For each $n \in \mathbb{N}$, the triplet $\left(\alpha_{n}, \beta_{n}, \gamma_{n}\right)$ is a triangle triplet.

Proof. We fix $n \in \mathbb{N}$ and take any $u_{1}, u_{3} \in\left[s, s_{n}\right], u_{2} \in\left[0,2 r_{n}\right], v_{2}, v_{3} \in\left[t, t_{n}\right], v_{1} \in\left[0,2 \rho_{n}\right]$. We first prove $\alpha_{n} \leq \beta_{n}+\gamma_{n}$. If $u_{2} \leq u_{3}$, then we have

$$
\alpha_{n} \leq F_{n}\left(u_{3},\left|v_{2}-v_{3}\right|\right) \leq F_{n}\left(u_{2}, v_{2}\right)+F_{n}\left(u_{3}, v_{3}\right)
$$

since $\left|v_{2}-v_{3}\right| \leq t_{n}-t \leq 2 \rho_{n}$ and $\left(u_{3}, u_{2}, u_{3}\right),\left(\left|v_{2}-v_{3}\right|, v_{2}, v_{3}\right)$ are triangle triplets. If $u_{2} \geq u_{3}$, then we see that

$$
s \leq u_{3} \leq u_{2} \leq 2 r_{n} \leq s_{n}
$$

By this, we have

$$
\alpha_{n} \leq F_{n}\left(u_{2},\left|v_{2}-v_{3}\right|\right) \leq F_{n}\left(u_{2}, v_{2}\right)+F_{n}\left(u_{3}, v_{3}\right) \text {. }
$$

These inequalities imply $\alpha_{n} \leq \beta_{n}+\gamma_{n}$. We have $\beta_{n} \leq \alpha_{n}+\gamma_{n}$ by the symmetric discussion as the proof of $\alpha_{n} \leq \beta_{n}+\gamma_{n}$. We next prove $\gamma_{n} \leq \alpha_{n}+\beta_{n}$. If $u_{3-i} \leq u_{i}$ and $v_{3-j} \leq v_{j}$ for $i, j=1,2$, then we have

$$
s \leq u_{i} \leq s_{n}, \quad t \leq v_{j} \leq t_{n}
$$

and then

$$
\gamma_{n} \leq F_{n}\left(u_{i}, v_{j}\right) \leq F_{n}\left(u_{1}, v_{1}\right)+F_{n}\left(u_{2}, v_{2}\right),
$$

which implies $\gamma_{n} \leq \alpha_{n}+\beta_{n}$. The proof is completed.

Choosing a subsequence of $n$, we can assume that there exist limits of $\left\{\alpha_{n}\right\}_{n \in \mathbb{N}},\left\{\beta_{n}\right\}_{n \in \mathbb{N}}$, and $\left\{\gamma_{n}\right\}_{n \in \mathbb{N}}$ as $n \rightarrow \infty$ and we denote these limits by $\alpha, \beta$, and $\gamma$ respectively. Note that $\alpha, \beta$, and $\gamma$ are positive, their triplet $(\alpha, \beta, \gamma)$ is a triangle triplet, and

$$
\alpha \leq F(s, 0), \quad \beta \leq F(0, t), \quad \gamma \leq F(s, t)-\eta .
$$

Indeed, for each $n$, it holds that

$$
\alpha_{n} \leq F_{n}(s, 0), \quad \beta_{n} \leq F_{n}(0, t), \quad \gamma_{n} \leq F_{n}\left(s_{n}, t_{n}\right) \leq F_{n}(s, t)-\eta .
$$

Define an mm-space $Z$ by

$$
Z:=\left(\left\{z_{00}, z_{10}, z_{01}, z_{11}\right\}, d_{Z}, \frac{1}{4} \sum_{i, j=0}^{1} \delta_{z_{i j}}\right),
$$

where $d_{Z}$ is a metric on $Z$ defined as

$$
\begin{aligned}
d_{Z}\left(z_{i j}, z_{i j}\right):=0, & d_{Z}\left(z_{i j}, z_{1-i, j}\right):=\alpha, \\
d_{Z}\left(z_{i j}, z_{i, 1-j}\right):=\beta, & d_{Z}\left(z_{i j}, z_{1-i, 1-j}\right):=\gamma
\end{aligned}
$$

for every $i, j=0,1$. Let us prove the following claim.

Claim 5.3. The sequence $\left\{X_{n} \times_{F_{n}} Y_{n}\right\}_{n \in \mathbb{N}}$ concentrates to $Z$ as $n \rightarrow \infty$. 
Proof. Let

$$
\begin{aligned}
S_{i}^{n}:=\left\{\bar{x}_{i}\right\} \times S^{k_{n}}\left(r_{n}\right) \subset X_{n}, & & \sigma_{i}^{n}:=\delta_{\bar{x}_{i}} \otimes \sigma^{k_{n}}, \\
T_{j}^{n}:=\left\{\bar{y}_{j}\right\} \times S^{l_{n}}\left(\rho_{n}\right) \subset Y_{n}, & & \tau_{j}^{n}:=\delta_{\bar{y}_{j}} \otimes \sigma^{l_{n}}, \\
\Omega_{i j}^{n}:=S_{i}^{n} \times T_{j}^{n} \subset X_{n} \times Y_{n}, & & \omega_{i j}^{n}:=\sigma_{i}^{n} \otimes \tau_{j}^{n}
\end{aligned}
$$

for every $i, j=0,1$, and let $p_{n}: X_{n} \times Y_{n} \rightarrow Z$ be the map defined by

$$
p_{n}(x):=z_{i j} \text { if } x \in \Omega_{i j}^{n} \text {. }
$$

Note that $p_{n \star}\left(m_{X_{n}} \otimes m_{Y_{n}}\right)=m_{Z}$. Let $\varepsilon>0$ be a sufficiently small arbitrary real number. We find a number $N \in \mathbb{N}$ such that

$$
\begin{aligned}
& \left|F_{n}(\varepsilon, \varepsilon)-F(\varepsilon, \varepsilon)\right|<\varepsilon, \quad\left|\alpha_{n}-\alpha\right|<\varepsilon, \quad\left|\beta_{n}-\beta\right|<\varepsilon, \quad\left|\gamma_{n}-\gamma\right|<\varepsilon, \\
& \operatorname{ObsDiam}\left(\left(S_{i}^{n},\|\cdot\|, \sigma_{i}^{n}\right) ;-\varepsilon\right)<\varepsilon, \quad \text { and } \quad \operatorname{ObsDiam}\left(\left(T_{j}^{n},\|\cdot\|, \tau_{i}^{n}\right) ;-\varepsilon\right)<\varepsilon
\end{aligned}
$$

hold for any $n \geq N$ and $i, j=0,1$. Let us prove that $p_{n}$ enforces $(72 F(\varepsilon, \varepsilon)+75 \varepsilon)$-concentration of $X_{n} \times F_{n} Y_{n}$ to $Z$ for any $n \geq N$. Fix $n$ with $n \geq N$. We first prove that $p_{n}$ is 1-Lipschitz up to $\varepsilon$. Take any $x_{0}, x_{0}^{\prime} \in S_{0}^{n}, x_{1}, x_{1}^{\prime} \in S_{1}^{n}$, $y_{0}, y_{0}^{\prime} \in T_{0}^{n}$, and $y_{1}, y_{1}^{\prime} \in T_{1}^{n}$. We see that

$$
\begin{aligned}
& s \leq\left\|x_{0}-x_{1}\right\| \leq s_{n}, \quad 0 \leq\left\|x_{i}-x_{i}^{\prime}\right\| \leq 2 r_{n}, \\
& t \leq\left\|y_{0}-y_{1}\right\| \leq t_{n}, \quad 0 \leq\left\|y_{j}-y_{j}^{\prime}\right\| \leq 2 \rho_{n} \text {. }
\end{aligned}
$$

Thus we have

$$
d_{F_{n}}\left(\left(x_{0}, y_{j}\right),\left(x_{1}, y_{j}^{\prime}\right)\right)=F_{n}\left(\left\|x_{0}-x_{1}\right\|,\left\|y_{j}-y_{j}^{\prime}\right\|\right) \geq \alpha_{n} \geq \alpha-\varepsilon=d_{Z}\left(z_{0 j}, z_{1 j}\right)-\varepsilon
$$

for every $j=0,1$, and

$$
d_{F_{n}}\left(\left(x_{i}, y_{0}\right),\left(x_{i}^{\prime}, y_{1}\right)\right)=F_{n}\left(\left\|x_{i}-x_{i}^{\prime}\right\|,\left\|y_{0}-y_{1}\right\|\right) \geq \beta_{n} \geq \beta-\varepsilon=d_{Z}\left(z_{i 0}, z_{i 1}\right)-\varepsilon
$$

for every $i=0,1$, and

$$
d_{F_{n}}\left(\left(x_{0}, y_{j}\right),\left(x_{1}, y_{1-j}\right)\right)=F_{n}\left(\left\|x_{0}-x_{1}\right\|,\left\|y_{0}-y_{1}\right\|\right) \geq \gamma_{n} \geq \gamma-\varepsilon=d_{Z}\left(z_{0 j}, z_{1,1-j}\right)-\varepsilon
$$

for every $j=0,1$. These imply that the map $p_{n}$ is 1-Lipschitz up to $\varepsilon$. By Lemma 4.9, we have

$$
p_{n}{ }^{\star} \operatorname{Lip}_{1}(Z) \subset U_{2 \varepsilon}\left(\mathcal{L i p}_{1}\left(X_{n} \times_{F_{n}} Y_{n}\right)\right) \text {. }
$$

We prove the other side inclusion. We take any function $f_{n} \in \mathcal{L} \operatorname{Lip}_{1}\left(X_{n} \times_{F_{n}} Y_{n}\right)$ and define a function $g_{n}: Z \rightarrow \mathbb{R}$ by

$$
g_{n}\left(z_{i j}\right):=\operatorname{lm}\left(f_{n} ; \omega_{i j}^{n}\right)
$$

for every $i, j=0,1$. By Lemma 2.26 and Lemma 3.22,

$$
\begin{aligned}
& \operatorname{LeRad}\left(\left(\Omega_{i j}^{n}, d_{F_{n}}, \omega_{i j}^{n}\right) ;-2 \varepsilon\right) \\
& \leq \operatorname{ObsDiam}\left(\left(\Omega_{i j}^{n}, d_{F_{n}}, \omega_{i j}^{n}\right) ;-2 \varepsilon\right) \\
& \leq 4 F_{n}\left(\operatorname{ObsDiam}\left(\left(S_{i}^{n},\|\cdot\|, \sigma_{i}^{n}\right) ;-\varepsilon\right), 0\right)+8 F_{n}\left(0, \operatorname{ObsDiam}\left(\left(T_{j}^{n},\|\cdot\|, \tau_{j}^{n}\right) ;-\varepsilon\right)\right) \\
& \leq 8 F_{n}(\varepsilon, \varepsilon)+16 F_{n}(\varepsilon, \varepsilon)=24 F_{n}(\varepsilon, \varepsilon) .
\end{aligned}
$$

Thus we have

$$
\begin{aligned}
& m_{X_{n}} \otimes m_{Y_{n}}\left\{z \in X_{n} \times Y_{n}|| f_{n}(z)-p_{n}{ }^{\star} g_{n}(z) \mid>24 F_{n}(\varepsilon, \varepsilon)\right\} \\
& =\frac{1}{4} \sum_{i, j=0}^{1} \omega_{i j}^{n}\left\{z \in \Omega_{i j}^{n}|| f_{n}(z)-\operatorname{lm}\left(f_{n} ; \omega_{i j}^{n}\right) \mid>24 F_{n}(\varepsilon, \varepsilon)\right\} \leq 2 \varepsilon,
\end{aligned}
$$


which implies $d_{\mathrm{KF}}^{m_{X_{n}} \otimes m_{Y_{n}}}\left(f_{n}, p_{n}{ }^{*} g_{n}\right)<24 F_{n}(\varepsilon, \varepsilon)+2 \varepsilon<24 F(\varepsilon, \varepsilon)+26 \varepsilon$.

Let us prove the following three inequalities. For every $i, j=0,1$,

$$
\begin{array}{r}
\left|\operatorname{lm}\left(f_{n} ; \omega_{0 j}^{n}\right)-\operatorname{lm}\left(f_{n} ; \omega_{1 j}^{n}\right)\right|<\alpha+48 F(\varepsilon, \varepsilon)+49 \varepsilon, \\
\left|\operatorname{lm}\left(f_{n} ; \omega_{i 0}^{n}\right)-\operatorname{lm}\left(f_{n} ; \omega_{i 1}^{n}\right)\right|<\beta+48 F(\varepsilon, \varepsilon)+49 \varepsilon, \\
\left|\operatorname{lm}\left(f_{n} ; \omega_{0 j}^{n}\right)-\operatorname{lm}\left(f_{n} ; \omega_{1,1-j}^{n}\right)\right|<\gamma+48 F(\varepsilon, \varepsilon)+49 \varepsilon .
\end{array}
$$

We define angles $\theta_{n}, \theta_{n}^{\prime}, \theta_{n}^{\prime \prime}, \phi_{n}, \phi_{n}^{\prime}, \phi_{n}^{\prime \prime} \in[0, \pi]$ by

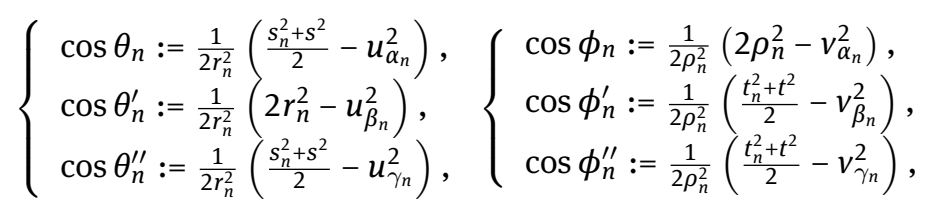

where $\left(u_{\alpha_{n}}, v_{\alpha_{n}}\right),\left(u_{\beta_{n}}, v_{\beta_{n}}\right)$, and $\left(u_{\gamma_{n}}, v_{\gamma_{n}}\right)$ are minimizers of $\alpha_{n}, \beta_{n}$ and $\gamma_{n}$ respectively, that is, $u_{\alpha_{n}}, u_{\gamma_{n}} \in$ $\left[s, s_{n}\right], u_{\beta_{n}} \in\left[0,2 r_{n}\right], v_{\beta_{n}}, v_{\gamma_{n}} \in\left[t, t_{n}\right], v_{\alpha_{n}} \in\left[0,2 \rho_{n}\right]$ such that

$$
F_{n}\left(u_{\alpha_{n}}, v_{\alpha_{n}}\right)=\alpha_{n}, F_{n}\left(u_{\beta_{n}}, v_{\beta_{n}}\right)=\beta_{n} \text {, and } F_{n}\left(u_{\gamma_{n}}, v_{\gamma_{n}}\right)=\gamma_{n} \text {. }
$$

In addition, we define rotations $\Theta_{n}, \Theta_{n}^{\prime}, \Theta_{n}^{\prime \prime}: \mathbb{R}^{k_{n}+1} \rightarrow \mathbb{R}^{k_{n}+1}$ by

$$
\begin{aligned}
& \Theta_{n}^{\star}\left(a_{1}, \ldots, a_{k_{n}+1}\right) \\
& :=\left(a_{1} \cos \theta_{n}^{\star}-a_{2} \sin \theta_{n}^{\star}, a_{1} \sin \theta_{n}^{\star}+a_{2} \cos \theta_{n}^{\star}, \ldots,\right. \\
& \left.\quad a_{k_{n}} \cos \theta_{n}^{\star}-a_{k_{n}+1} \sin \theta_{n}^{\star}, a_{k_{n}} \sin \theta_{n}^{\star}+a_{k_{n}+1} \cos \theta_{n}^{\star}\right)
\end{aligned}
$$

for $\left(a_{1}, \ldots, a_{k_{n}+1}\right) \in \mathbb{R}^{k_{n}+1}$, where $\left(\Theta_{n}^{\star}, \theta_{n}^{\star}\right)$ is each of $\left(\Theta_{n}, \theta_{n}\right),\left(\Theta_{n}^{\prime}, \theta_{n}^{\prime}\right)$, and $\left(\Theta_{n}^{\prime \prime}, \theta_{n}^{\prime \prime}\right)$, and we recall that $k_{n}$ is odd. We also define rotations $\Phi_{n}, \Phi_{n}^{\prime}, \Phi_{n}^{\prime \prime}: \mathbb{R}^{l_{n}+1} \rightarrow \mathbb{R}^{l_{n}+1}$ by the same way as above $\Theta_{n}^{\star}$, that is,

$$
\begin{aligned}
\Phi_{n}^{\star} & \left(b_{1}, \ldots, b_{l_{n}+1}\right) \\
:= & \left(b_{1} \cos \phi_{n}^{\star}-b_{2} \sin \phi_{n}^{\star}, b_{1} \sin \phi_{n}^{\star}+b_{2} \cos \phi_{n}^{\star}, \ldots,\right. \\
& \left.b_{l_{n}} \cos \phi_{n}^{\star}-b_{l_{n}+1} \sin \phi_{n}^{\star}, b_{l_{n}} \sin \phi_{n}^{\star}+b_{l_{n}+1} \cos \phi_{n}^{\star}\right) .
\end{aligned}
$$

We prove (5.2). Fix $j \in\{0,1\}$. Let $T_{n}: \Omega_{0 j}^{n} \rightarrow \Omega_{1 j}^{n}$ be the map defined by

$$
T_{n}\left(\left(\bar{x}_{0}, a\right),\left(\bar{y}_{j}, b\right)\right):=\left(\left(\bar{x}_{1}, \Theta_{n}(a)\right),\left(\bar{y}_{j}, \Phi_{n}(b)\right)\right)
$$

for $a \in S^{k_{n}}\left(r_{n}\right), b \in S^{l_{n}}\left(\rho_{n}\right)$. Note that $T_{n \star} \omega_{0 j}^{n}=\omega_{1 j}^{n}$. For any $a \in S^{k_{n}}\left(r_{n}\right), b \in S^{l_{n}}\left(\rho_{n}\right)$, we have

$$
\begin{aligned}
& \left\|\left(\bar{x}_{0}, a\right)-\left(\bar{x}_{1}, \Theta_{n}(a)\right)\right\|=\sqrt{s^{2}+2 r_{n}^{2}\left(1-\cos \theta_{n}\right)}=u_{\alpha_{n}}, \\
& \left\|\left(\bar{y}_{j}, b\right)-\left(\bar{y}_{j}, \Phi_{n}(b)\right)\right\|=\sqrt{2 \rho_{n}^{2}\left(1-\cos \phi_{n}\right)}=v_{\alpha_{n}},
\end{aligned}
$$

so that for any $z \in \Omega_{0 j}^{n}$,

$$
d_{F_{n}}\left(z, T_{n}(z)\right)=F_{n}\left(u_{\alpha_{n}}, v_{\alpha_{n}}\right)=\alpha_{n} .
$$

The measure (id, $\left.T_{n}\right)_{\star}\left(\omega_{0 j}^{n}\right)$ is an $\alpha_{n}$-transport plan between $\omega_{0 j}^{n}$ and $\omega_{1 j}^{n}$. By Lemma 2.27 and Lemma 3.22, we have

$$
\begin{aligned}
& \left|\operatorname{lm}\left(f_{n} ; \omega_{0 j}^{n}\right)-\operatorname{lm}\left(f_{n} ; \omega_{1 j}^{n}\right)\right| \\
& \leq \alpha_{n}+\sum_{i=0}^{1} \operatorname{ObsDiam}\left(\left(\Omega_{i j}^{n}, d_{F_{n}}, \omega_{i j}^{n}\right) ;-2 \varepsilon\right) \leq \alpha_{n}+\sum_{i=0}^{1} 24 F_{n}(\varepsilon, \varepsilon) \\
& <\alpha+48 F(\varepsilon, \varepsilon)+49 \varepsilon .
\end{aligned}
$$


Thus (5.2) is obtained. By means of similar arguments, one can prove (5.3) and (5.4). Indeed, one can use maps $T_{n}^{\prime}: \Omega_{i 0}^{n} \rightarrow \Omega_{i 1}^{n}$ and $T_{n}^{\prime \prime}: \Omega_{0 j}^{n} \rightarrow \Omega_{1,1-j}^{n}$ for $i, j \in\{0,1\}$ defined by

$$
\begin{aligned}
& T_{n}^{\prime}\left(\left(\bar{x}_{i}, a\right),\left(\bar{y}_{0}, b\right)\right):=\left(\left(\bar{x}_{i}, \Theta_{n}^{\prime}(a)\right),\left(\bar{y}_{1}, \Phi_{n}^{\prime}(b)\right)\right), \\
& T_{n}^{\prime \prime}\left(\left(\bar{x}_{0}, a\right),\left(\bar{y}_{j}, b\right)\right):=\left(\left(\bar{x}_{1}, \Theta_{n}^{\prime \prime}(a)\right),\left(\bar{y}_{1-j}, \Phi_{n}^{\prime \prime}(b)\right)\right)
\end{aligned}
$$

for $a \in S^{k_{n}}\left(r_{n}\right), b \in S^{l_{n}}\left(\rho_{n}\right)$.

Combining (5.2) - (5.4) and Lemma 4.8 implies $d_{\mathrm{KF}}^{m_{Z}}\left(g_{n}\right.$, $\left.\mathcal{L i p} p_{1}(Z)\right)<48 F(\varepsilon, \varepsilon)+49 \varepsilon$. Thus we have

$$
d_{\mathrm{KF}}^{m_{X_{n}} \otimes m_{Y_{n}}}\left(f_{n}, p_{n}{ }^{*} \mathcal{L i p}_{1}(Z)\right) \leq d_{\mathrm{KF}}^{m_{X_{n}} \otimes m_{Y_{n}}}\left(f_{n}, p_{n}{ }^{*} g_{n}\right)+d_{\mathrm{KF}}^{m_{Z}}\left(g_{n}, \mathcal{L i} p_{1}(Z)\right)<72 F(\varepsilon, \varepsilon)+75 \varepsilon .
$$

Therefore the map $p_{n}$ enforces $(72 F(\varepsilon, \varepsilon)+75 \varepsilon)$-concentration of $X_{n} \times_{F_{n}} Y_{n}$ to $Z$ for every $n \geq N$. By Theorem 2.19, the sequence $\left\{X_{n} \times_{F_{n}} Y_{n}\right\}_{n \in \mathbb{N}}$ concentrates to $Z$ as $n \rightarrow \infty$. The proof of the claim is now completed.

Since

$$
\alpha \leq F(s, 0), \quad \beta \leq F(0, t), \quad \text { and } \quad \gamma \leq F(s, t)-\eta,
$$

the mm-space $X \times_{F} Y$ is not mm-isomorphic to $Z$. Thus Claim 5.3 means that the condition (1) of Theorem 1.1 does not hold. Therefore we obtain "(1) $\Rightarrow$ (2)" of Theorem 1.1.

Remark 5.4. In the above proof of Theorem 1.1, if there exist finite limits of both $\left\{s_{n}\right\}_{n \in \mathbb{N}}$ and $\left\{t_{n}\right\}_{n \in \mathbb{N}}$, then the three sequences $\left\{\alpha_{n}\right\}_{n \in \mathbb{N}},\left\{\beta_{n}\right\}_{n \in \mathbb{N}}$, and $\left\{\gamma_{n}\right\}_{n \in \mathbb{N}}$ converge without taking a subsequence and these limits are

$$
\alpha=\min _{\substack{s \leq u_{1} \leq s_{\infty} \\ 0 \leq v_{1} \leq 2 \rho}} F\left(u_{1}, v_{1}\right), \quad \beta=\min _{\substack{0 \leq u_{2} \leq 2 r \\ t \leq v_{2} \leq t_{\infty}}} F\left(u_{2}, v_{2}\right), \quad \gamma=\min _{\substack{s \leq u_{3} \leq s_{\infty} \\ t \leq v_{3} \leq t_{\infty}}} F\left(u_{3}, v_{3}\right),
$$

where

$$
s_{\infty}:=\lim _{n \rightarrow \infty} s_{n}, t_{\infty}:=\lim _{n \rightarrow \infty} t_{n}, r:=\frac{\sqrt{s_{\infty}^{2}-s^{2}}}{2}, \rho:=\frac{\sqrt{t_{\infty}^{2}-t^{2}}}{2}
$$

\section{Product of $\boldsymbol{N}$ metric measure spaces}

In this section, we consider the concentration of product spaces of $N \mathrm{~mm}$-spaces. Indeed, we generalize Theorem 1.1 to the following.

Theorem 6.1. Let $F_{n}, F \in \mathscr{F}^{N}, n=1,2, \ldots$ Assume that $F_{n}$ converges pointwise to $F$ as $n \rightarrow \infty$. Then the following conditions are equivalent to each other.

(1) For any $N$ sequences $\left\{X_{n}^{i}\right\}_{n \in \mathbb{N}}, i=1, \ldots, N$, of mm-spaces concentrating to mm-spaces $X^{i}$ respectively, the sequence

$$
\left\{\left(\prod_{i=1}^{N} X_{n}^{i}, d_{F_{n}}, \otimes_{i=1}^{N} m_{X_{n}^{i}}\right)\right\}_{n \in \mathbb{N}}
$$

of their product spaces concentrates to the product space

$$
\left(\prod_{i=1}^{N} X^{i}, d_{F}, \otimes_{i=1}^{N} m_{X^{i}}\right)
$$

as $n \rightarrow \infty$.

(2) For any $\left(s_{1}, \ldots, s_{N}\right) \in[0,+\infty)^{N}$,

$$
\lim _{n \rightarrow \infty}\left(F_{n}\left(s_{1}, \ldots, s_{N}\right)-\inf _{s_{i} \leq s_{i}^{\prime}} F_{n}\left(s_{1}^{\prime}, \ldots, s_{N}^{\prime}\right)\right)=0 .
$$

(3) For any $D>0$,

$$
\lim _{n \rightarrow \infty} \sup _{0 \leq s_{i} \leq D}\left(F_{n}\left(s_{1}, \ldots, s_{N}\right)-\inf _{s_{i} \leq s_{i}^{\prime}} F_{n}\left(s_{1}^{\prime}, \ldots, s_{N}^{\prime}\right)\right)=0
$$


We denote by $\left(\prod_{i=1}^{N} X^{i}\right)_{p}$ the $l_{p}$-product spaces of $X^{1}, \ldots, X^{N}$, which are generated by

$$
F_{p}^{N}\left(s_{1}, \ldots, s_{N}\right):= \begin{cases}\left(\sum_{i=1}^{N} s_{i}^{p}\right)^{\frac{1}{p}} & \text { if } p<+\infty \\ \max _{i=1, \ldots, N} s_{i} & \text { if } p=+\infty\end{cases}
$$

Lemma 6.2. Let $p \in[1,+\infty]$ and let $X^{1}, \ldots, X^{N}$ be $N$ mm-spaces. Then we have

$$
\operatorname{ObsDiam}\left(\left(\prod_{i=1}^{N} X^{i}\right)_{p} ;-\sum_{i=1}^{N} \kappa_{i}\right) \leq \operatorname{ObsDiam}\left(X^{1} ;-\kappa_{1}\right)+2 \sum_{i=2}^{N} \operatorname{ObsDiam}\left(X^{i} ;-\kappa_{i}\right)
$$

for any $\kappa_{1}>0$ and any $\kappa_{2}, \ldots, \kappa_{N} \in(0,1 / 2)$.

Lemma 6.3. Let $F \in \mathscr{F}^{N}$ and let $X^{1}, \ldots, X^{N}$ be $N$ mm-spaces. Then

$$
\operatorname{ObsDiam}\left(\left(\prod_{i=1}^{N} X^{i}, d_{F}, \bigotimes_{i=1}^{N} m_{X^{i}}\right) ;-2 \sum_{i=1}^{N} \kappa_{i}\right) \leq 4 F^{1}\left(\operatorname{ObsDiam}\left(X^{1} ;-\kappa_{1}\right)\right)+8 \sum_{i=2}^{N} F^{i}\left(\operatorname{ObsDiam}\left(X^{i} ;-\kappa_{i}\right)\right) .
$$

for any $\kappa_{1}>0$ and any $\kappa_{2}, \ldots, \kappa_{N} \in(0,1 / 4)$, where $F^{i}:=F \circ \iota_{i}$ and $\iota_{i}:[0,+\infty) \rightarrow[0,+\infty)^{N}$ is the natural $i$-th inclusion map.

Proof of Lemma 6.2. Since the $l_{p}$-product has the iterated property

$$
\left(\prod_{i=1}^{N} X^{i}\right)_{p}=\left(\prod_{i=1}^{N-1} X^{i}\right)_{p} \times_{p} X^{N}
$$

by Lemma 3.26, we have

$$
\begin{aligned}
& \operatorname{ObsDiam}\left(\left(\prod_{i=1}^{N} X^{i}\right)_{p} ;-\sum_{i=1}^{N} \kappa_{i}\right) \\
& \leq \operatorname{ObsDiam}\left(\left(\prod_{i=1}^{N-1} X^{i}\right)_{p} ;-\sum_{i=1}^{N-1} \kappa_{i}\right)+2 \operatorname{ObsDiam}\left(X^{N} ;-\kappa_{N}\right) .
\end{aligned}
$$

We obtain (6.1) by induction.

Proof of Lemma 6.3. In the same way as the proof of Lemma 3.27, since $F \leq \sum_{i=1}^{N} F^{i}:=G$ and the mm-space $\left(\prod_{i=1}^{N} X^{i}, d_{G}, \otimes_{i=1}^{N} m_{X^{i}}\right)$ is mm-isomorphic to

$$
\left(\prod_{i=1}^{N}\left(X^{i}, F^{i} \circ d_{X^{i}}, m_{X^{i}}\right)\right)_{1},
$$

by Lemma 6.2, we have (6.2).

Proof of Theorem 6.1. We can prove Theorem 6.1 by imitating the proof of Theorem 1.1. We obtain the implication from (2) to (3) by imitating Lemmas 4.6 and 4.10. In the proof of the implication from (3) to (1), the key to imitate is an estimate of the observable diameter of the product space. We have already obtained Lemma 6.3, so that we obtain this implication. Let us prove the implication from (1) to (2). Assume that the condition (2) does not hold. Up to choosing a subsequence of $n$, we can assume that there exist a real numbers $\eta>0$, an $N$-tuple $\left(s^{1}, \ldots, s^{N}\right) \in[0,+\infty)$ and a sequence $\left\{\left(s_{n}^{1}, \ldots, s_{n}^{N}\right)\right\}_{n \in \mathbb{N}} \subset[0,+\infty)$ such that

$$
s^{i}<s_{n}^{i} \quad \text { and } \quad F_{n}\left(s^{1}, \ldots, s^{N}\right)>F_{n}\left(s_{n}^{1}, \ldots, s_{n}^{N}\right)+\eta
$$

for any $n \in \mathbb{N}$ and every $i$. Let $X^{i}, i=1, \ldots, N$, be the $N$ mm-spaces defined by

$$
X^{i}:=\left(\left\{x_{0}^{i}, x_{1}^{i}\right\}, d_{X^{i}}, \frac{1}{2} \delta_{x_{0}^{i}}+\frac{1}{2} \delta_{x_{1}^{i}}\right), \quad d_{X^{i}}\left(x_{0}^{i}, x_{1}^{i}\right):=s^{i} .
$$


We consider the $N$ mm-spaces $X_{n}^{i}$ defined by

$$
X_{n}^{i}:=X^{i} \times_{2}\left(S^{k_{n}^{i}}\left(r_{n}^{i}\right),\|\cdot\|, \sigma^{k_{n}^{i}}\right),
$$

where $r_{n}^{i}:=\sqrt{\left(s_{n}^{i}\right)^{2}-\left(s^{i}\right)^{2}} / 2$ and $k_{n}^{i}:=2 \max \left\{n,\left\lceil\left(r_{n}^{i}\right)^{4}\right\rceil\right\}+1$. By imitating the proof of Claim 5.3, we see that $\left\{\left(\prod_{i=1}^{N} X_{n}^{i}, d_{F_{n}}, \otimes_{i=1}^{N} m_{X_{n}^{i}}\right)\right\}_{n \in \mathbb{N}}$ has a subsequence that does not concentrate to $\left(\prod_{i=1}^{N} X^{i}, d_{F}, \otimes_{i=1}^{N} m_{X^{i}}\right)$. Thus we obtain the implication from (1) to (2). The proof is completed.

Example 6.4. The following function is an example of metric preserving functions that are not of iterated type.

$$
F_{\text {cyc }}\left(s_{1}, s_{2}, s_{3}\right):=\max \left\{s_{1}+s_{2}, s_{2}+s_{3}, s_{3}+s_{1}\right\} .
$$

$F_{\text {cyc }}$ does not have the iterated property like $F_{p}^{N}$. Theorem 6.1 can be applied to such functions.

Acknowledgement: The author would like to thank Professor Takashi Shioya, Hiroki Nakajima, and Shinichiro Kobayashi for their comments and encouragement, and the referees for their careful reading and kind comments. He is also grateful to Professor Takumi Yokota and Professor Ryunosuke Ozawa for their information and comments.

The author was supported by JSPS KAKENHI (No.17J02121).

Conflict of interest: The author states no conflict of interest.

\section{References}

[1] J. Borsík and J. Doboš, On a product of metric spaces, Math. Slovaca 31 (1981), no. 2, 193-205 (English, with Russian summary).

[2] J. Doboš and Z. Piotrowski, Some remarks on metric preserving functions, Real Anal. Exchange 19 (1993/94), no. 1, 317-320.

[3] K. Funano and T. Shioya, Concentration, Ricci curvature, and eigenvalues of Laplacian, Geom. Funct. Anal. 23 (2013), no. 3, 888-936.

[4] M. Gromov, Metric structures for Riemannian and non-Riemannian spaces, Reprint of the 2001 English edition, Modern Birkhäuser Classics, Birkhäuser Boston, Inc., Boston, MA, 2007.

[5] D. Kazukawa, R. Ozawa, and N. Suzuki, Stabilities of rough curvature dimension condition, J. Math. Soc. Japan 72 (2020), no. 2, 541-567.

[6] J. L. Kelley, General topology, D. Van Nostrand Company, Inc., Toronto-New York-London, 1955.

[7] M. Kuczma, An introduction to the theory of functional equations and inequalities, 2nd ed., Birkhäuser Verlag, Basel, 2009. Cauchy's equation and Jensen's inequality; Edited and with a preface by Attila Gilányi.

[8] M. Ledoux, The concentration of measure phenomenon, Mathematical Surveys and Monographs, vol. 89, American Mathematical Society, Providence, RI, 2001.

[9] P. Lévy, Problèmes concrets d'analyse fonctionnelle. Avec un complément sur les fonctionnelles analytiques par F. Pellegrino, Gauthier-Villars, Paris, 1951 (French). 2d ed.

[10] V. D. Milman, The heritage of P. Lévy in geometrical functional analysis, Astérisque 157-158 (1988), 273-301. Colloque Paul Lévy sur les Processus Stochastiques (Palaiseau, 1987).

[11] H. P. Mulholland, On generalizations of Minkowski's inequality in the form of a triangle inequality, Proc. London Math. Soc. (2) 51 (1950), 294-307.

[12] T. Neubrunn and T. Šalát, Über eine Klasse metrischer Räume, Acta Fac. Natur. Univ. Comenian 10 (1965), no. fasc. 3, 23-30 (1965) (German, with Slovak and Russian summaries).

[13] R. Ozawa and T. Shioya, Estimate of observable diameter of $l_{p}$-product spaces, Manuscripta Math. 147 (2015), no. 3-4, 501-509.

[14] R. Ozawa and N. Suzuki, Stability of Talagrand's inequality under concentration topology, Proc. Amer. Math. Soc. 145 (2017), no. 10, 4493-4501.

[15] R. Ozawa and T. Yokota, Stability of RCD condition under concentration topology, Calc. Var. Partial Differential Equations 58 (2019), no. 4, Paper No. 151, 30.

[16] M. Petrík, New solutions to Mulholland inequality, Aequationes Math. 89 (2015), no. 4, 1107-1122.

[17] T. Shioya, Metric measure geometry, IRMA Lectures in Mathematics and Theoretical Physics, vol. 25, EMS Publishing House, Zürich, 2016. Gromov's theory of convergence and concentration of metrics and measures. 